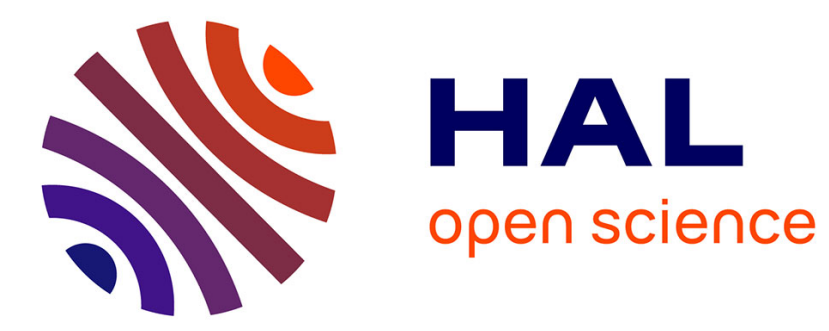

\title{
Psychologie individuelle et stabilité d'un équilibre général concurrentiel dans le Traité d'économie pure de Maurice Allais
}

Jean-Sébastien Lenfant

\section{- To cite this version:}

Jean-Sébastien Lenfant. Psychologie individuelle et stabilité d'un équilibre général concurrentiel dans le Traité d'économie pure de Maurice Allais. Revue Economique, 2005, Revue économique, 56 (4), pp.855-888. 10.3917/reco.564.0855 . hal-01712704

\author{
HAL Id: hal-01712704 \\ https://hal.science/hal-01712704
}

Submitted on 19 Feb 2018

HAL is a multi-disciplinary open access archive for the deposit and dissemination of scientific research documents, whether they are published or not. The documents may come from teaching and research institutions in France or abroad, or from public or private research centers.
L'archive ouverte pluridisciplinaire $\mathbf{H A L}$, est destinée au dépôt et à la diffusion de documents scientifiques de niveau recherche, publiés ou non, émanant des établissements d'enseignement et de recherche français ou étrangers, des laboratoires publics ou privés.

\section{(이) $\$$}

Distributed under a Creative Commons Attribution - NonCommercial - NoDerivatives| 4.0 
Ce document est un preprint d'un article publié. Il peut contenir des changements mineurs par rapport à la version publiée. Référence pour citation :

Lenfant, J. S. (2005). Psychologie individuelle et stabilité d'un équilibre général concurrentiel dans le Traité d'économie pure de Maurice Allais. Revue économique, 56(4) : 855-888.

DOI : $10.3917 /$ RECO.564.0855

\title{
PSYCHOLOGIE INDIVIDUELLE ET STABILITÉ D'UN EQUILIBRE GÉNÉRAL CONCURRENTIEL DANS LE TRAITÉ D'ÉCONOMIE PURE DE MAURICE ALLAIS
}

\section{Jean-Sébastien Lenfant*}

\begin{abstract}
Résumé. - Cet article analyse la contribution de Maurice Allais à l'étude de la stabilité d'un équilibre concurrentiel dans le Traité d'économie pure (1943). Les commentateurs retiennent essentiellement qu'il a proposé, le premier, des conditions suffisantes de stabilité équivalentes à l'bypothèse de substituabilité brute (Negishi, 1962). Le projet théorique de Allais est en fait bien plus ambitieux car il entend fonder la propriété de stabilité sur des hypothèses conformes à l'introspection et concilier ainsi le réalisme des hypothèses et la rigueur logique de la démonstration. Le but de cet article est de faire ressortir les fondements psychologiques de la théorie de l'équilibre général selon. Allais et d'en discuter la portée.
\end{abstract}

\section{PSYCHOLOGY AND THE STABILITY OF A GENERAL COMPETITIVE EQUILIBRIUM IN MAURICE ALLAIS'S TRAITÉ D'ÉCONOMIE PURE}

Summary. - This article analyses Maurice Allais's contribution to the study of the stability of a competitive equilibrium. In his Traite d'économie pure (1943), be demonstrates the local stability of a walrasian economy under a set of hypothesis tantamount to gross substitutability (Negishi, 1962). However, it would be unfair to reduce his contribution to this set of sufficient conditions. Allais's purpose is much more ambitious, to the extent that he endeavours to ground stability on a set of introspectively appealing assumptions, built on a detailed and sophisticated theory of choice. He aims at conciliating the realism of assumptions and the logical rigor of the demonstration. In this article, we highlight the psychological foundations of general equilibrium analysis in Allais's thought.

Maurice Allais - Théorie de l'équilibre général - Théorie des choix Substituabilité - Stabilité

Maurice Allais - General equilibrium theory - choice theory Substitutability - Stability

Classification JEL : B21, B31.

\footnotetext{
* Jean-Sébastien Lenfant, GRESE, Maison des Sciences Economiques, 106-112 Bd de l'Hôpital, 75647 Paris cédex 13 ; e-mail: lenfant@univ-paris1.fr. L'auteur remercie tout particulièrement Jérôme Lallement et Marion Gaspard ainsi que deux rapporteurs anonymes de la revue. Une première version de cet article a fait l'objet d'une présentation au Colloque de l'Association Française de Science Economique en septembre 2000.
} 


\section{PSYCHOLOGIE INDIVIDUELLE ET STABILITÉ D'UN EQUILIBRE GÉNÉRAL CONCURRENTIEL DANS LE TRAITÉ D'ÉCONOMIE PURE DE MAURICE AlLAIS}

\section{INTRODUCTION}

Il est aujourd'hui reconnu que Maurice Allais a proposé la première démonstration de stabilité locale d'une économie d'échange walrasienne en 1943 dans son ouvrage $A$ la recherche d'une discipline économique, réédité ultérieurement sous le titre Traité d'économie pure (désormais Traitê). Les commentateurs qui ont tenté un bilan des apports de Maurice Allais à la théorie économique, après qu'il a obtenu le prix à la mémoire d'Alfred Nobel en 1988, montrent deux attitudes différentes en ce qui concerne cette contribution pionnière. Pour certains, l'analyse de la stabilité d'une économie walrasienne ne semble pas faire partie des contributions significatives de Allais (Drèze, 1989). Allais lui-même (Allais, 1997, 4) dans son allocution de réception du prix Nobel devant l'Académie Royale des Sciences de Suède, retient essentiellement de cet ouvrage la démonstration d'équivalence entre les deux théorèmes fondamentaux de l'économie du bien-être ${ }^{1}$. Pour d'autres au contraire (Grandmont, 1989, 29 ; Munier, 1989, 5) ${ }^{2}$, Allais a proposé une analyse pionnière de la stabilité d'un équilibre concurrentiel - obtenant des conditions comparables à l'hypothèse de substituabilité brute ${ }^{3}-$, et sa démonstration s'appuierait implicitement sur la seconde méthode de Lyapounov (1907). Allais, en 1943, anticiperait donc de seize ans la démonstration de stabilité globale par Arrow, Block et Hurwicz (1959). Plus nuancé, Negishi (1962) insiste pour sa part sur l'originalité de la démarche et il signale que Allais "fit des hypothèses qui, prises ensemble sont essentiellement les mêmes que celle de substituabilité brute" (Negishi, 1962, 656) ${ }^{4}$. Il y a donc manifestement sur ce point précis de la stabilité d'un système walrasien une appréciation changeante de l'apport de Maurice Allais. Weintraub (1991a et 1991b) a identifié une partie de la difficulté que soulève la thèse de Grandmont et Munier. Elle concerne l'attribution de l'emploi de la seconde méthode de Lyapounov à Allais. Il a montré ce qu'un tel jugement contient de reconstruction rétrospective ${ }^{5}$ et comment il oriente la représentation de l'évolution de la théorie économique.

L'objet de cet article est d'opérer un déplacement dans la manière d'appréhender la contribution de Allais. L'hypothèse de substituabilité brute est couramment jugée peu satisfaisante. On devrait donc s'étonner que Allais mette en avant, non seulement l'originalité de sa démarche, mais aussi la généralité de son résultat ${ }^{6}$ :

La démonstration que nous avons donnée de la stabilité de l'équilibre est la première à notre connaissance qui ait été faite dans un cas suffisamment général. En tout cas, notre marche d'approche est, pensons-nous, absolument originale. (Allais, 1993, Introduction à la deuxième édition, 25)

${ }^{1}$ Voir également Allais (1978, deuxième partie) et Allais $(1989,34)$

2 Voir aussi Belloc et Moreaux (1987)

${ }^{3}$ Rappelons que l'hypothèse de "substituabilité brute" dit que la demande excédentaire de chaque bien est une fonction croissante du prix de tous les autres biens (Arrow, Block et Hurwicz, 1959, 86).

${ }^{4}$ Voir aussi Negishi $(1958,445 n)$.

${ }^{5}$ Il reste que comme l'a remarqué Negishi $(1962,656)$ la démonstration de la stabilité de Allais peut être correctement reformulée dans les termes de la seconde méthode de Lyapounov.

${ }^{6}$ voir aussi Allais $(1978,34)$ 
Autrement dit, la question de savoir si Allais fit des hypothèses équivalentes à la substituabilité brute ne se réduit pas à sa dimension technique ou mathématique. Au-delà de la démonstration de la stabilité elle-même, précisément analysée par Weintraub, c'est le projet théorique d'ensemble du Traité qui doit être pris en compte pour appréhender la place de Maurice Allais dans l'histoire de la théorie de l'équilibre général. Ainsi, à la fin du chapitre III du Traité consacré à la stabilité ("la dynamique du déséquilibre"), Allais estime qu'il est " bautement probable qu'à structure constante, l'Économie évoluerait sous l'action de mobiles qui caractérisent le comportement des individus et des entreprises vers un état d'équilibre et que cet état d'équilibre serait stable" (Allais, 1993, 503). Allais est donc soucieux de concilier, autant que possible, l'exigence logique de la démonstration et le réalisme des hypothèses.

Nous voyons alors qu'il est trompeur de prêter à Allais l'antériorité d'un ensemble de conditions mathématiques suffisantes pour la stabilité de l'équilibre, parce qu'il a cherché en fait, plus fondamentalement, à fournir un ensemble d'hypothèses empiriquement et/ou psychologiquement vraisemblables de la stabilité d'un équilibre général concurrentiel. Comment ces hypothèses sont-elles obtenues et validées? Certes, c'est là une question intrigante et presque incongrue, aujourd'hui, après les résultats de Sonnenschein (1973), Mantel (1974) et Debreu (1974) sur les propriétés des fonctions de demande excédentaire. Mais c'est aussi, selon nous, l'un des aspects méconnus et tout à fait originaux du Traité ${ }^{7}$.

Dans cet article, nous nous proposons en premier lieu de faire ressortir la structure des arguments qui conduisent au résultat de stabilité dans le Traité et, en second lieu, d'en discuter la cohérence et les limites, Allais étant d'ailleurs le premier à reconnaittre qu'il ne peut fournir une "démonstration rigoureuse complète" (Allais, 1993, 271). Nous montrerons que pour Allais, les propriétés de stabilité des économies walrasiennes devraient trouver leur raison d'être, en partie tout au moins, dans les données psychologiques individuelles qui sous-tendent la formation des offres et des demandes individuelles et agrégées. C'est là précisément que se joue l'articulation du réalisme des hypothèses et de la rigueur du raisonnement visée par Allais pour donner des fondements microéconomiques à la stabilité. L'effort analytique de Allais pour relier stabilité et préférences individuelles est sans doute l'un des aspects les plus originaux du Traité et permet, selon nous, de mieux interpréter les méthodes et hypothèses retenues. Cela témoigne aussi, dès 1943, des difficultés d'une telle entreprise.

Dans une première section (1. De l'économie réelle à l'introspection : la méthode de Maurice Allais), nous discutons les sources de la théorie des choix dans le Traité. La place essentielle qui est réservée aux données psychologiques et empiriques singularise Allais. Tout en s'appuyant sur la tradition parétienne, Allais propose une représentation originale des préférences et des choix individuels sur des lois psychologiques données ou confortées par l'expérience. Dans une deuxième section (2. Préférences, demande et stabilité), nous faisons apparaitre les traits les plus saillants de sa typologie des biens substituts et complémentaires et l'usage qui en est fait pour fonder une hypothèse d'indépendance psychologique entre les biens et conduire l'analyse de la stabilité de l'équilibre général. Dans une troisième et dernière section (3. Du bon usage de la psychologie dans le Traité), nous proposons une typologie des hypothèses impliquées dans la construction d'ensemble du Traité et nous les évaluons au regard des principes méthodologiques revendiqués par Allais.

\footnotetext{
${ }^{7}$ La recherche de fondements microéconomiques est bien plus superficielle dans V aleur et capital, même si Hicks parvient à des résultats proches de ceux de Allais .
} 


\section{DE L’ÉCONOMIE RÉELLE A L'INTROSPECTION : LA MÉTHODE DE MAURice Allais}

L'objectif de cette section est de préciser comment Allais se positionne par rapport aux théoriciens de l'équilibre général et notamment par rapport à l'évolution de la théorie des choix dans les années 1930. Des facteurs très divers d'ordre historique et intellectuel font du Traité un ouvrage en décalage par rapport à l'histoire de la théorie du choix et de l'équilibre général. Nous commencerons par replacer le Traité dans son contexte de rédaction et d'influences (1.1. Les sources du Traité). Puis nous verrons comment Allais défend une utilisation raisonnée du modèle d'équilibre général comme approximation du phénomène économique (1.2. La synthèse de l'abstraction et de l'introspection). Finalement, nous soulignerons la place de premier ordre qu'occupent les explications psychologiques dans la théorie de l'équilibre général selon Allais (1.3. Les fondements psychologiques de la théorie de l'équilibre général).

\subsection{Les sources du Traité}

Le Traité occupe une place à part dans le développement de la théorie économique, pour des raisons diverses qui tendent à se renforcer mais qu'il est important de distinguer. En effet, la pensée économique de Maurice Allais s'est façonnée en marge de certains développements théoriques qui auraient pu infléchir sa présentation de la théorie de l'équilibre général. Mais dans les faits, cette marginalité est partiellement volontaire et assumée. Quelques précisions sur la genèse et les sources du Traité permettront de mieux apprécier la spécificité du projet de Allais ${ }^{8}$.

Le Traité se veut avant tout l'œuvre d'un ingénieur pour les ingénieurs, un "exposé systématique et cohérent du mécanisme d'ensemble de l'économie" (Allais, 1943, 15 ; 1993, 17) qui livrera "des principes d'action formant une doctrine cohérente" (Allais, 1943, 16; 1993, 19). Selon le plan initial, $A$ la recherche d'une discipline économique devait être composé de trois parties : la première consacrée à la théorie de l'équilibre général en économie pure, la seconde consacrée à l'économie réelle, et la troisième à l'économie normative. Dans les faits, l'ouvrage publié en 1943 ne correspond qu'à la première partie et constitue aujourd'hui le Traité d'économie pure.

Jusqu'à la guerre, Allais ne s'intéresse pas particulièrement à l'économie. C'est seulement à partir de juillet 1940 qu'il prend connaissance des ouvrages de Walras, Fisher, et Pareto (Allais, 1997, 3) et de la plus grande partie des ouvrages cités dans le Traité. D'un point de vue analytique, l'ouvrage le plus proche du Traité et dont la lecture fut décisive est sans conteste le Manuel de Pareto et particulièrement son

\footnotetext{
8 Pendant la guerre, Maurice Allais est ingénieur des Mines à Nantes. De janvier 1941 à juillet 1943 , il travaille à la rédaction acharnée d'un ouvrage de plus de 900 pages. Son ambition alors est "de poser les fondements sur lesquels il serait possible de construire une politique économique et sociale" (Allais, 1997, 3) et plus précisément "de trouver une solution au problème fondamental de toute économie, à savoir comment promouvoir le plus grand rendement réalisable tout en garantissant une distribution du revenu globalement acceptable" (Allais, 1997, 3). Le plus urgent est donc d'établir une synthèse théorique mais aussi de concilier le "tableau réconfortant" qu'offre la théorie pure et "l'analyse décevante de la réalité" (Allais, 1943, 18; 1993, 20). D’un point de vue normatif, le Traité doit faire éclater l'opposition simpliste entre laissez-faire ou libéralisme d'une part, et planisme ou corporatisme de l'autre, et leur substituer une troisième voie, "un tiers chemin: l'organisation de la liberté économique dans le cadre de la loi"' (Allais, 1943, 19 ; 1993, 21).
} 
Appendice (Pareto, 1909, 539-671) 9. Le Manuel de Pareto sera donc pour nous un point de comparaison précieux sur tous les sujets qui sous-tendent l'étude de la stabilité : la théorie des choix et de la demande.

A cela, il faut ajouter les nombreuses lectures d'économistes français, ou traduits en langue française, qui ont contribués à répandre et à faire connaitre l'économie mathématique dans les années 1920 et 1930. Les références les plus saillantes à cet égard sont Divisia (1928), Bousquet (1930) et, dans une mesure moindre, Colson (1924). Allais a très certainement assisté au cours de Divisia à l'Ecole Polytechnique dans l'année $1931-1932^{10}$, et il a pris connaissance également de ses autres cours et de son ouvrage Économique rationnelle. Ses réflexions sur la place de l'introspection en économie lui ont vraisemblablement donné des motifs de justification de sa théorie des choix. Quant aux Institutes de science économique de Bousquet (1930-1936), Allais leur doit davantage une perception des limites de la théorie de l'équilibre général walrasien et la conscience du risque d'une abstraction qui déconnecte la théorie de ses visées d'application (Allais, 1993, 869). Enfin, le Cours de Colson discute largement les comportements d'offre et de demande, en insistant sur les comportements de substitution (Colson, 1924, 214-224), qui occupent bien évidemment une place centrale dans l'exposé de Allais.

Du point de vue de la théorie des choix et de l'équilibre général, il faut remarquer que très peu de textes étrangers figurent dans le Traité. Allais n'a eu connaissance, au moment de la rédaction, ni de Valeur et Capital de Hicks (1939), ni des articles de Samuelson (1941 et 1942) sur la stabilité. La seule contribution en anglais à laquelle il soit fait référence est l'article de Hicks et Allen de 1934 : “A Reconsideration of the Theory of Value". Parmi les ouvrages mentionnés qui accordent une place importante à la théorie des préférences, de l'utilité, de la demande et des prix, il faut signaler également l'opuscule Théorie mathématique de la valeur en régime de libre concurrence (Hicks, 1937) ainsi que De l'utilité (Roy, 1942). Quelles conclusions pouvons-nous en tirer? En un sens, compte tenu des circonstances de la guerre et de la place privilégiée accordée aux économistes français, la construction théorique de Allais s'est faite dans l'ignorance de certains développements de la théorie de la demande et de l'équilibre général dans le monde anglo-saxon (les articles de Samuelson sur les "préférences révélées" et l'utilité (Samuelson, 1938a et 1938b), ceux sur la stabilité (Samuelson, 1941 et 1942)). Mais ce jugement doit immédiatement être nuancé. Si l'on considère en effet la somme théorique que représentent l'article de Hicks et Allen (1934) et le fascicule en français de Hicks (1937) - deux références connues de Allais - du point de vue de la théorie de l'utilité, de la demande, et de la stabilité, alors il faut considérer que les choix accomplis par Allais sont volontairement en décalage par rapport à l'orientation de la révolution ordinaliste et à l'étude de la stabilité à la Hicks. En gardant à l'esprit ces remarques historiques, nous pouvons apprécier l'originalité des positions méthodologiques de Allais, notamment en matière de théorie des choix.

\footnotetext{
9 “C'est à partir des très nombreuses réflexions que m'a suscitées ma lecture en avril 1941 de l'Appendice du Manuel d'Economie Politique (p. 539-671) de Vilfredo PARETO que j'ai pu élaborer l'architecture de mon ouvrage de 1943, et mes développements sur la satisfaction ordinale et la satisfaction absolue [utilité cardinale], sur l'équilibre du consommateur et de l'entreprise, sur les fonctions de demande, sur les conditions de stabilité, et surtout sur le concept de surplus distribuable qui constitue un des piliers essentiels de mon ouvrage de 1943 et de toute mon œuvre ultérieure." (Allais, 1993, Introduction à la troisième édition, 30)

10 Divisia est associé nommément à la dédicace du Traité: "A mes maittres LeON Walras, Vilfredo Pareto, Irving Fisher, FrançoIS Divisia, et aux camarades de ma génération à qui il revient de construire l'Economie de demain"' (Allais, 1943, 2).
} 


\subsection{La synthèse de l'abstraction et de l'introspection}

Du fait de ses ambitions opérationnelles affichées, Allais est tenu de concilier étroitement deux impératifs. L'ensemble des phénomènes économiques dont il entend faire la synthèse rend nécessaires de nombreuses abstractions théoriques. La difficulté est de trouver une forme d'abstraction qui puisse être développée mathématiquement sans déformer la représentation du fonctionnement d'une économie réelle :

Notre modèle schématique ne constitue qu'une économie irréelle, très éloignée de la réalité, mais, par le fait même que cette économie reproduira d'une manière extrêmement simplifiée les caractères fondamentaux de l'économie réelle, elle permettra de définir, de manière précise, des concepts, dont la transposition à cette économie se fera ensuite d'elle-même. Le cadre mathématique dont nous nous servirons a donc pour seul objet de constituer un support commode pour la pensée et pour le raisonnement; il n'a nullement pour but la représentation de la réalité. (Allais, 1943, 60 ; 1993, 64)

Pour mener de front l'ambition de rigueur et celle du réalisme, Allais met en place une forme assez complexe de validation des énoncés. Un énoncé de théorie économique peut soit porter sur le comportement observable des agents, soit sur les données subjectives et inobservables des préférences. Conformément à cette distinction, la validation des énoncés économiques peut se faire de deux manières complémentaires: soit par la confrontation de ces énoncés avec "l'expérience quotidienne" des comportements individuels, soit par la confrontation des hypothèses initiales dont ces énoncés sont les conséquences et de l'intuition que nous en avons, même approximative ${ }^{11}$.

La validation des énoncés économiques fonctionne donc à deux niveaux dans le Traité, et on peut y voir le point de départ de ce qui fait l'originalité de Allais par rapport au courant ordinaliste. Allais appuie en effet sa démarche sur une synthèse entre le modèle des sciences expérimentales et les données d'une science de l'introspection. Par anticipation de ce qui va suivre, on peut dire que la référence au modèle des sciences expérimentales le place dans le courant ordinaliste, tandis que la référence aux données de l'introspection prend chez lui une dimension qui va franchement à contre-courant du mouvement ordinaliste dans les années 1930 et $1940{ }^{12}$.

Certes, comme Pareto l'avait déjà explicité, ce n'est que dans la confrontation avec l'expérience que se décide la valeur de la représentation abstraite (Allais, 1943, 60 ; 1993, 64). Toutefois, à la différence de Hicks et Allen (1934), Allais est particulièrement attaché à la valeur introspective des hypothèses initiales de la théorie pure. Sur ce sujet, il cite longuement l'ouvrage de Divisia, Économique rationnelle. Dans cet ouvrage, l'articulation de la théorie à l'expérience n'est pas l'occasion d'un rapprochement trivial avec la physique ; au contraire, il s'y joue la spécificité de la science économique. Divisia écrit en effet :

Dans les sciences physiques, l'explication scientifique tend à substituer à la contemplation des phénomènes concrets, mais complexes, qui nous entourent, la considération de phénomènes plus simples, mais beaucoup plus abstraits (...). Il y a là, certes, un attrait puissant qui tente sans cesse le savant, mais qui ne peut le plus souvent lui procurer le repos de l'esprit.

\footnotetext{
11 Voir par exemple ce que dit Allais de l'hypothèse de prévision parfaite (Allais, 1943, 56 ; 1993, 61).

12 Une étude plus précise, que nous ne pouvons entreprendre ici, conduirait certainement à distinguer le projet ordinaliste tel qu'il est mis en œuvre par Allen, Hicks et Samuelson d'une part et les principes méthodologiques plus complexes que l'on trouve chez Pareto ou Slutsky, qui n'évacuent pas radicalement le recours à l'introspection ou à la psychologie.
} 
Dans les sciences morales, il n'en est pas de même; la matière sur laquelle nous travaillons, c'est, au fond, nous-mêmes, si bien que l'explication scientifique, au lieu de nous éloigner constamment du sujet de notre observation, nous en rapproche au contraire : quand elle est parvenue à nous faire considérer un phénomène comme la conséquence de mobiles spécifiquement humains, ces derniers nous apparaissent comme trop évidents, trop naturels, trop nécessaires pour que nous soyons tentés d'y voir autre chose que la manifestation de vérités premières, qui ne nécessitent plus d'explication. (Divisia, 1928, 105, cité par Allais, 1943, 38-9 ; 1993, 37)

Il faut prendre la mesure des enjeux d'une telle affirmation. En reprenant à son compte la thèse de Divisia, Allais ne s'en remet pas exclusivement à l'observation des faits pour décider de la validité d'une théorie ou d'une hypothèse. Il revendique aussi une place pour l'intuition ou l'introspection (Allais ne fait pas une distinction claire entre ces deux termes). Certes l'intuition (ou l'introspection) ne dispense pas d'une analyse rigoureuse, mais elle n'est pas dénuée d'un certain pouvoir de jugement :

Tandis que dans les sciences physiques la confrontation des théories avec la réalité se réduit à la comparaison des résultats prévus par le calcul avec ceux donnés par l'observation, dans les sciences morales, et en Économie en particulier, une telle confrontation résultera tout autant de la comparaison directe des hypothèses de départ avec les données immédiates de notre intuition. (Allais, 1943, 39 ; 1993, 37, nos italiques)

La valeur de jugement reconnue à l'introspection (ou à l'intuition) à égalité avec l'observation, recèle des conséquences méthodologiques radicales parce qu'elle fait apparaittre deux sources possibles de justification. Toute la question est alors de savoir quelle place Allais accorde à l'une et à l'autre et comment nous devons interpréter ce "tout autant". Allais suggère en fait qu'un compromis est possible entre le souci de réalisme des hypothèses, d'une part, et la visée d'instrumentalisation des modèles, d'autre part ${ }^{13}$. La validation d'un énoncé d'observation peut tenir à la fois à la pertinence empirique de cet énoncé et à la justesse des hypothèses dont il est déduit, sans que l'on sache si un mode de validation peut l'emporter sur un autre. Il semble bien en effet qu'une certaine liberté est laissée au théoricien pour jouer sur un registre de validation plutôt que sur un autre. En définitive, Allais laisse entrevoir une forme progressive et assez souple de validation des énoncés. Leur validation expérimentale sera d'autant plus forte que les hypothèses initiales à partir desquelles ces énoncés sont obtenus auront reçu le soutien de l'introspection ${ }^{14}$. Mais il se peut aussi que des énoncés acceptables ou raisonnables sur les comportements observables viennent conforter les choix d'hypothèses restrictives ou approximatives sur les préférences. Ainsi, chez Allais, induction et déduction se côtoient intimement, selon une modalité circulaire d'aller et retour entre les données de l'introspection et les déductions expérimentales dont on trouve les principes chez Pareto (1909, 574), mais également dans les ouvrages précédemment cités de Divisia (1928, 361-363) et de Bousquet (1930, 72-73) ${ }^{15}$.

Quoi qu'il en soit, on doit s'attendre à ce que l'introspection joue un rôle très important dans l'exposé des propriétés des comportements de demande et par extension, dans celles d'une économie walrasienne.

13 Voir Allais $(1989,45)$.

14 Voir également Allais $(1989,44)$.

${ }^{15}$ L'objet de cet article n'est pas d'établir une comparaison systématique et fine des méthodes de Hicks et de Allais. On notera simplement que Hicks, sans renoncer complètement à des arguments introspectifs, leur donne toujours un poids faible dans son argumentation, conformément à une attitude méthodologique globalement méfiante à l'égard des arguments hédonistes. 


\subsection{Les fondements psychologiques de la théorie de l'équilibre général}

La stabilité de l'équilibre général repose essentiellement sur les comportements de marchés, qui dépendent à leur tour de comportements individuels fondés sur un ensemble d'hypothèses relatives aux préférences des individus. Compte tenu des recommandations méthodologiques de Allais, il n'est pas étonnant que le Traité réserve une place très importante à une analyse approfondie des préférences individuelles fondée sur les données de l'introspection psychologique.

Nous touchons ici à une différence de taille entre Allais et les acteurs de la "révolution ordinaliste", que nous n'avons fait qu'évoquer jusqu'ici. Allais croit profondément aux fondements psychologiques des lois du comportement individuel et à la possibilité de déduire des lois précises du comportement à partir de lois psychologiques conformes à l'introspection. On l'a dit, il connait l'article de Hicks et Allen (1934) ainsi que l'opuscule en français de Hicks (1937), qui montrent le degré de généralité auquel peut parvenir la théorie du choix à partir d'un ensemble restreint d'hypothèses, en raisonnant en termes ordinaux. Qu'il ne s'engage pas dans cette voie est un acte délibéré. Pour autant, son rapport à la théorie ordinaliste, de Pareto à Hicks, n'est pas simple. Sans nier la généralité à laquelle conduit l'approche ordinale, Allais est soucieux avant tout de conserver à la théorie de l'utilité un fondement introspectif. Il y a là une position subtile qu'il faut éclairer.

En fait, la rupture avec le mouvement ordinaliste se joue sans doute à un niveau plus profond. Pour Fisher et Pareto, la psychologie, comme physique des émotions et des sensations, doit rester en dehors de la théorie économique. L'objet de leur refus correspond en fait à la version psychophysique introduite par Edgeworth (Chaigneau, 1998). Allais affirme au contraire que cette perspective psychophysique doit être préservée : "Il n'est pas impossible que l'on réalise dans l'avenir en psychologie économique des expériences analogues à celles de la psychologie physiologique" (Allais, 1943, 159) ${ }^{16}$.

La structure argumentative du Traité devient alors plus claire. Allais va recourir à des arguments hédonistes et introspectifs, en plus d'arguments d'expérience, pour sélectionner des profils de préférence dominants qui lui permettront de valider, in fine, l'hypothèse d'indépendance psychologique entre les biens, hypothèse dont la traduction mathématique est une fonction d'utilité additive et séparable. Tout le raisonnement analytique sera alors conduit à partir d'une telle fonction, au moins à titre d'approximation, et au voisinage d'un point ${ }^{17}$. Les principes ordinalistes sont alors réintroduits dans la mesure où n'importe quelle transformée croissante de cette fonction pourrait servir à décrire les préférences individuelles.

Allais identifie donc sa théorie des préférences individuelles à la "représentation concrète des psychologies individuelles" (Allais, 1943, 61 ; 1993, 67), conduite par "l'introspection psychologique" et "confirmée par l'expérience la plus courante" (Allais, 1943, 115 ; 1993, 120) :

Nous essaierons d'établir quelles propriétés l'on peut par simple introspection psychologique attribuer à ces surfaces [d'indifférence]. Ces propriétés permettront ultérieurement de déduire certaines lois économiques que l'on peut considérer comme établies par l'expérience. Elles se trouveront alors

16 Toute la section consacrée aux satisfactions absolues (\$\$68-70) est imprégnée par cette idée. Allais fait ainsi référence à Weber et Fechner, ainsi qu'à l'ouvrage Psychologie expérimentale de Pieron (Allais, 1993, 163)

17 C'est-à-dire une fonction pour laquelle la dérivée de l'utilité marginale est négative (utilité marginale décroissante) et très grande par rapport aux différentes dérivées secondes croisées de la fonction d'utilité. Cette propriété, bien sûr, n'a aucune raison d'être vérifiée pour toute transformation croissante de la fonction d'utilité. 
pleinement confirmées. (Allais, 1943, 61, nos italiques ; 1993, 67)

Ici, très nettement, l'interprétation de la méthodologie du Traité va dans le sens d'un cheminement harmonieux entre deux pôles: les données psychologiques fondamentales données par l'introspection sont confortées par les énoncés observables, conformes à l'expérience, qui peuvent s'en déduire.

Mais surtout, il faut y voir une ambition centrale du Traité qui va au-delà de la théorie du choix. En effet, lorsqu'il aborde la question de la stabilité d'une économie d'échange, Allais met en avant le rôle des préférences individuelles; il fait reposer la théorie de l'équilibre général sur des fondements psychologiques.

L'économie walrasienne sans production, affirme-t-il, n'est “qu'une image théorique extraordinairement simplifiée de l'Économie réelle" (Allais, 1943, 486 ; 1993, 472) et elle rend d'autant plus délicate l'interprétation des résultats et leur validation "empirique". Pourtant, les propriétés heuristiques de cette représentation simplifiée restent intactes. Notamment, le fait de ne pas prendre en considération la production dans l'analyse "ne change rien au mécanisme général de l'évolution du marché" (Allais, 1993, 471) et Allais y voit un argument supplémentaire pour focaliser l'attention sur les préférences individuelles. Autrement dit, l'analyse psychologique des préférences individuelles irrigue toute la construction du Traité, à tel point que Allais présente son projet comme une recherche de fondements psychologiques de l'équilibre général, dont les implications porteraient jusque dans l'analyse de sa stabilité : "Finalement, on voit que ce sont les psychologies individuelles qui constituent la base même de l'évolution du marché" (Allais, 1943, 486 ; 1993, 471).

Notre objectif, à présent, est de mettre à l'épreuve ce projet et donc de faire ressortir les hypothèses essentielles sur les préférences et de préciser leur justification et leurs implications pour la demande et la stabilité d'un équilibre général.

\section{Préférences, Demande et Stabilité}

A partir d'une typologie des couples de biens et de services Allais souhaite déduire des lois de demande qui permettront d'engager l'étude de la stabilité. L'idée essentielle qui permet d'organiser l'analyse est que dans l'ensemble, les biens peuvent être considérés comme psychologiquement indépendants les uns par rapport aux autres. Dans cette section, notre objectif est de présenter l'architecture analytique du Traité depuis les fondations psychologiques des préférences jusqu'à la démonstration de la stabilité d'une économie d'échange walrasienne. C'est alors seulement, dans une troisième section, que nous pourrons mettre à l'épreuve la méthode et le raisonnement de Allais. Nous présentons d'abord la typologie complexe des couples de biens et de services (2.1. La typologie des couples de biens et de services). Cette construction permet à Allais de privilégier une hypothèse générale d'indépendance psychologique entre les biens et de lui associer certains comportements de demande (2.2. L'hypothèse d'indépendance psychologique et les lois de la demande). Enfin, ces comportements sont à leur tour insérés dans l'analyse de la stabilité d'une économie d'échange walrasienne (2.3. La stabilité de l'économie walrasienne)

\subsection{La typologie des couples de biens et de services}

Formellement, la théorie des courbes d'indifférence et des cartes d'indifférence présente de nombreuses affinités avec l'Appendice mathématique du Manuel de 
Pareto, mais Allais en revendique les fondements introspectifs. Ainsi, le principe de la convexité des courbes d'indifférence est "établi par notre introspection psychologique" (Allais, 1943, 122 ; 1993, 125). Il exprime que la désirabilité relative (notre taux marginal de substitution) d'un bien 1 décroît, à satisfaction constante, lorsque la quantité consommée du bien 2 augmente. De même, mais avec moins d'assurance, Allais $(1943,123)$ pense que "dans la plupart des cas" le taux marginal de substitution du bien 2 au bien 1 augmente lorsque, à quantité de bien 1 fixée, on augmente la quantité que l'agent économique détient en bien 2. C'est la "loi des désirabilités relatives décroissantes" (Allais, 1943, 123) ${ }^{18}$. Elle n'est plus vérifiée pour deux biens dans une relation hiérarchique, comme le beurre et la margarine (Allais, 1943, 124). Ces quelques propriétés des courbes d'indifférence sont fournies par l'introspection psychologique. Elles servent de fondement à l'analyse de la substituabilité, c'est-à-dire à la classification des biens selon qu'ils sont substituts ou complémentaires ${ }^{19}$, sans doute la plus pointue dans toute la littérature économique (Allais, 1993, $\$ \$ 64-65,132-154)^{20}$.

Nous rentrons dans le cœur de l'analyse de la substituabilité lorsqu'il est question de réaliser une classification des "couples de services". Toute la typologie des biens substituts et complémentaires découle de la considération des cas intermédiaires entre les complémentaires parfaits (ou "services associés" (Allais, 1943, 128 ; 1993, 132)) et les substituts parfaits (ou "services homologues" (Allais, $1943,130 ; 1993,132)$ ). Formellement, la typologie des biens complémentaires et substituts, chez Allais, repose sur la forme des lignes de satiété, c'est-à-dire des courbes frontières le long desquelles l'utilité marginale d'un des biens est nulle, en prenant pour repère, dans le plan des biens, le point de satiété absolue, c'est-à-dire l'intersection des courbes de satiété relative à chaque bien. Sa classification s'apparente donc, dans l'esprit tout au moins, à celles proposées antérieurement par Johnson, Allen, et Friedman et elle suppose un point de satiété ${ }^{21}$. Cela donne le sentiment d'un retour en arrière par rapport aux développements des années 1930 et en même temps d'une synthèse impressionnante des réflexions de Fisher et Pareto sur les courbes d'indifférence ${ }^{22}$.

Soit $\mathrm{M}_{\mathrm{M}}$ le point de satiété dans le plan $\mathrm{OAB}$, et $\mathrm{Q}_{1}$ le quadrant situé au sudouest de $\mathrm{M}_{\mathrm{M}}, \mathrm{Q}_{2}$ celui situé au sud-est et $\mathrm{Q}_{3}$ celui situé au nord-ouest. Soit $\gamma_{A}$ et $\gamma_{B}$ les lignes de satiété relatives respectivement au bien A et B. La classification découle des positions relatives des lignes de satiété dans ces quadrants. Allais n'en

\footnotetext{
${ }_{18}$ Ce n'est pas pour simplifier la lecture, dans la mesure où le lecteur est familiarisé avec l'hypothèse du taux marginal de substitution décroissant, qui correspond à la propriété précédente, et qui exprime les variations du TMS le long d'une courbe d'indifférence.

19 En 1943, Allais parle de "substitualité". Dans l'édition de 1993 du Traité, le terme devient "substituabilité".

20 Allais ne cachera pas d'ailleurs l'importance qu'il accorde à cette question de la substituabilité, ce qui en fait un moment essentiel de son projet théorique : "Nous considérons l'analyse complète de la classification des couples de services] comme une des plus difficile de l'économique théorique. En fait, nous ne sommes arrivé aux définitions qui suivent qu'après des mois de tâtonnement et de réflexion" (Allais, 1993, 132, note 1). Ce temps de gestation est loin d'être négligeable, rapporté au temps de rédaction du Traité.

21 Allais $(1943,127-8)$ fait des hypothèses plus simples sur les lignes de satiété, qui impliquent que deux biens complémentaires restent complémentaires quelle que soit la quantité possédée de chacun de ces biens à l'intérieur des courbes frontières. Techniquement, cela revient à exclure les cas particuliers envisagés par Friedman (1934) dans lesquels la courbe de satiété par rapport à un bien peut être d'abord croissante puis décroissante. Historiquement, on trouve déjà une analyse en termes de lignes de satiété chez Lenoir (1913) (voir Chaigneau et Le Gall, 1998). Pour ce qui concerne Johnson (1913), il ne raisonne pas sur les lignes de satiété, mais Allen (1934) introduit cet outil pour généraliser la classification de Johnson.

22 La puissance de la typologie géométrique de Allais apparaît notamment lorsqu'il introduit l'articulation des goûts et des besoins. Les besoins sont matérialisés par les lignes de satiété, tandis que les goûts le sont par les cartes d'indifférence. Un besoin peut être complètement satisfait sans que la satisfaction ait atteint son maximum. Voir notamment Allais $(1943,151)$.
} 
retient que deux ${ }^{23}$.

Dans le cas où les courbes de satiété sont dans le quadrant $\mathrm{Q}_{1}$ (figure 1), les services (A) et (B) sont "complémentaires psychologiques" (Allais, 1943, 130 ; 1993, 133).

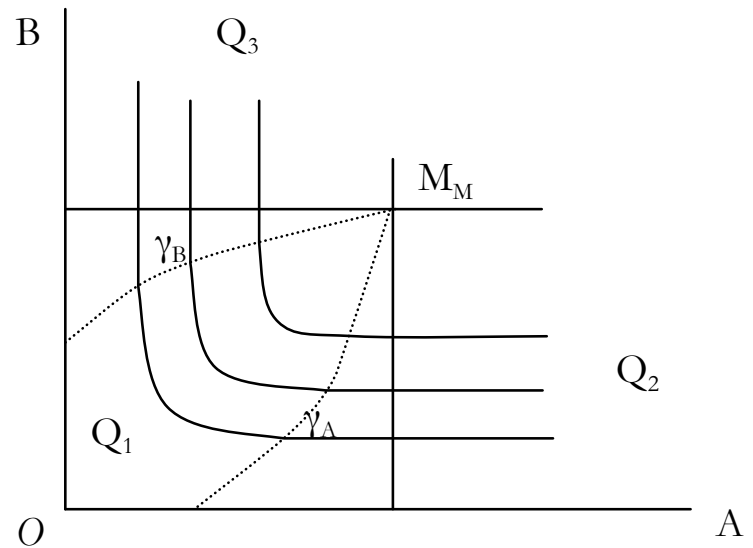

Figure 1

Complémentaires psychologiques.

D’après Allais (1943, 130, fig.3)

Dans le cas où les lignes de satiété sont dans les quadrants $\mathrm{Q}_{2}$ et $\mathrm{Q}_{3}$ (figure 2), les services (A) et (B) sont "substituts psychologiques" (Allais, 1943, $130 ; 1993,133$ ) ${ }^{24}$.

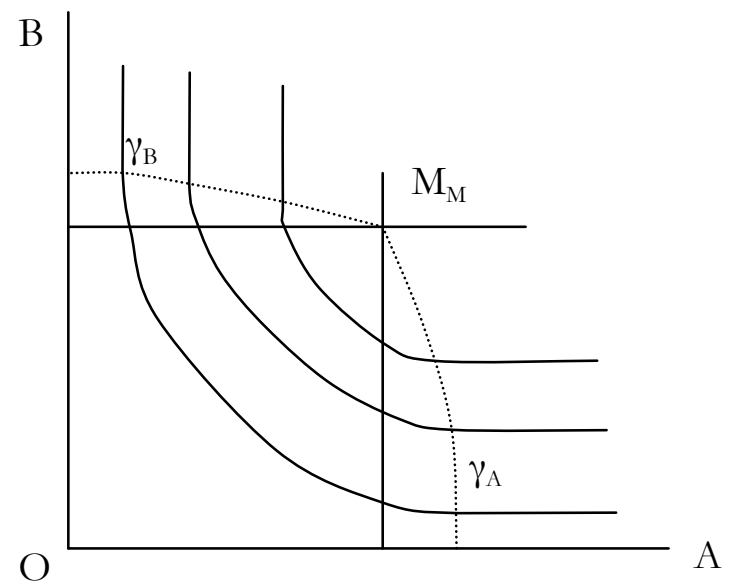

Figure 2

Substituts psychologiques

D’après Allais (1943, 131, fig.4)

Entre les services complémentaires et substituts, on trouve les services indépendants. Idéalement, pour de tels services, la satiété d'un bien n'est pas affectée par la modification de la quantité de l'autre. Les lignes de satiété $\gamma_{B}$ et $\gamma_{A}$

${ }^{23}$ En effet, il juge que le cas pour lequel une et une seule courbe de satiété se trouve dans le quadrant sudouest et l'autre dans le quadrant $\mathrm{Q}_{2}$ ou $\mathrm{Q}_{3}$ "semble sinon impossible tout au moins extrêmement rare, et elle nous paraît pouvoir être négligée” (Allais, 1943, 130; 1993, 133). Allais (1943, 131 ; 1993, 133, note 2) justifie d'exclure ce cas particulier par le fait qu'il n'en a pas trouvé d'exemple, conformément aux principes inductifs qu'il a adoptés.

${ }^{24}$ En d'autres termes, pour deux complémentaires, le niveau de satiété de l'un augmente avec la quantité de l'autre (pain et vin, sel et viande, huîtres et vin blanc), pour deux substituts, le niveau de satiété de l'un diminue avec l'augmentation de la quantité de l'autre (théâtre et cinéma, thé et café, vin blanc et vin rouge). 
sont parallèles aux axes $\mathrm{OA}$ et $\mathrm{OB}$. Lorsque les lignes de satiété sont proches de ce cas idéal, on parlera de biens peu dépendants.

Allais affine encore sa typologie en identifiant des configurations particulières à l'intérieur de chaque classe ${ }^{25}$. Deux d'entre elles nous intéressent particulièrement à l'intérieur de la classe des biens substituts. Une première configuration exprime le fait que deux biens substituts peuvent être dans une relation hiérarchique l'un par rapport à l'autre. On parlera alors de biens "substituts asymétriques" (Allais, 1943, 134 ; 1993, 136). La deuxième configuration importante envisagée par Allais concerne les "services à substituabilité symétrique accusée", c'est-à-dire lorsque deux biens présentent un fort degré de substituabilité. Géométriquement, les lignes de satiété forment alors un angle très ouvert. Au nom de l'expérience - et non plus de l'introspection - Allais suggère que les courbes d'indifférence auront en ce cas une très faible courbure (figure 3).

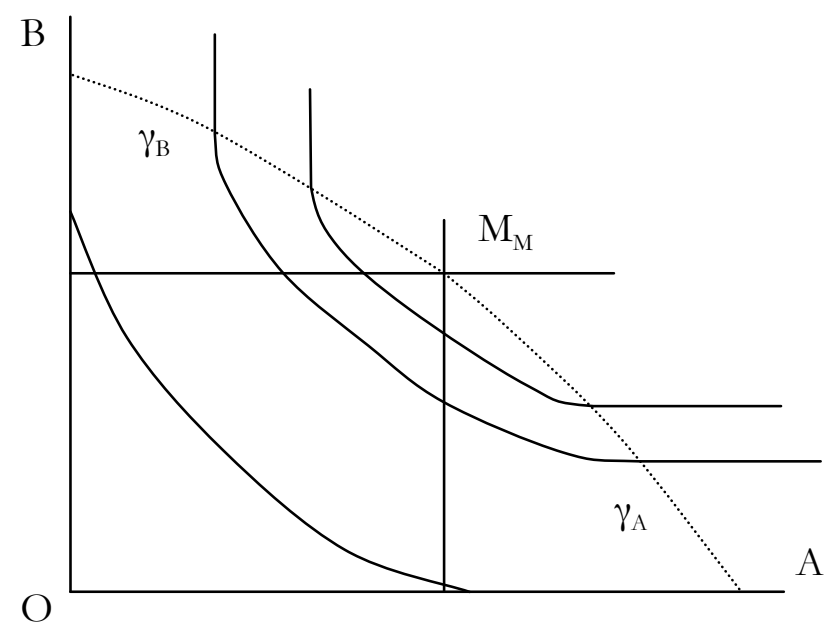

Figure 3

\section{Lignes d'indifférence dans le cas de biens substituts symétriques accusés. Source : Allais (1943, 136 ;1993, 137, fig. 10)}

Quels enseignements peut-on tirer de cette analyse psycho-géométrique? Elle permet d'établir une relation de correspondance entre la continuité psychologique présumée des préférences (on passe insensiblement des complémentaires parfaits aux substituts parfaits) et la continuité géométrique de leurs représentation (la seconde illustrant la première), continuité entre le cas des biens complémentaires parfaits, indépendants, et substituts parfaits ${ }^{26}$.

Il nous reste alors à saisir dans quel but Allais a élaboré une telle classification, en insistant à ce point sur la continuité des relations de substituabilité, et en

25 Ainsi dans le cas de biens complémentaires, on peut identifier la relation entre un bien principal et un bien accessoire. La satiété du bien principal (par exemple les journées de sport d'hiver) est peu affectée par la quantité consommée du bien accessoire (les billets de téléphérique), tandis que la quantité limite du bien accessoire est proportionnelle à la quantité consommée du bien principal. De même, dans le cas de biens indépendants, on peut identifier des situations où les biens sont "semi indépendants" (Allais, 1943, 133 ; 1993 , 135).

26 "On s'aperçoit alors comment on peut passer insensiblement et par transitions continues du cas des services indépendants au cas des services homologues" (Allais, 1943, 136 ; 1993, 137). Si, dans le cas de substituts accusés, les lignes d'indifférence étaient très coudées, il y aurait une solution de continuité entre la substituabilité accusée et les substituts parfaits. Tout se passe donc comme si l'on pouvait passer directement et sans autre détour introspectif, des cartes d'indifférence aux angles des lignes de satiété aux points de satisfaction maxima, et comme si la forme de ces courbes d'indifférence était toute entière contenue dans la connaissance de cet angle au point de satiété absolue $\mathrm{M}_{\mathrm{M}}$. 
prenant soin d'écarter certaines situations embarrassantes, voire inclassables. Ce but ne tarde pas à apparaître. Il s'agit en fait de justifier l'hypothèse générale d'indépendance psychologique entre les biens comme approximation des caractéristiques d'un système économique et d'en déduire les comportements de demande les plus attendus. C'est le moment où les données d'expérience ou d'observation viennent compléter les énoncés que l'on peut déduire des hypothèses psychologiques.

\subsection{L'hypothèse d'indépendance psychologique et les lois de la demande}

Dans le Traité, Allais retient l'hypothèse d'indépendance psychologique (ou de faible dépendance psychologique) entre les biens comme une approximation raisonnable de la réalité. L'hypothèse d'indépendance psychologique signifie que, étant donnés deux biens ou services distincts, en général, le niveau de saturation de la consommation de chaque bien est indépendant de la quantité détenue ou consommée de l'autre bien (lignes de satiété parallèles aux axes). Cette hypothèse doit servir de support à l'analyse du comportement de demande et finalement, à l'étude de la stabilité. Au terme de l'exposé géométrique des préférences, Allais retient essentiellement une division du plan entre trois zones correspondant à trois types principaux de positions relatives des lignes de satiété et, donc, de dépendance psychologique: les biens complémentaires accusés, les substituts accusés et les biens faiblement dépendants. C'est une typologie grossière et réductrice parce qu'elle restreint fortement les positions relatives des lignes de satiété envisageables et parce qu'elle focalise l'attention sur des cas particuliers. C'est aussi l'occasion pour Allais d'introduire l'hypothèse de faible dépendance psychologique, qui deviendra finalement l'hypothèse d'indépendance psychologique ${ }^{27}$ :

L'introspection psychologique semble bien montrer que la plupart des services peuvent être considérés comme peu dépendants, les substituts et les complémentaires accusés ne constituant que des cas statistiquement exceptionnels. (Allais, 1943, $143 ; 1993,145$ )

Allais ramène donc toutes les configurations possibles du champ de préférences à trois situations types. Toute la stratégie argumentative qui se met alors en place vise à montrer que les situations extrêmes de substituts accusés et de complémentaires accusés peuvent se ramener elles aussi, analytiquement, au cas simple d'indépendance psychologique. L'hypothèse d'une fonction d'utilité additivement séparable servira alors de référence pour les développements ultérieurs. Toute cette démarche de validation de l'hypothèse d'indépendance psychologique est conduite pas à pas, et Allais s'appuie pour cela aussi bien sur des données introspectives que sur des faits d'expérience qu'il pense généralisables à l'ensemble des biens et services. Il nous faut donc faire un détour par les lois de demande pour prendre toute la mesure de l'hypothèse d'indépendance psychologique.

Dans le Traité, l'exposé des lois de demande (\$\$169-177) fait partie intégrante du chapitre III (Dynamique du déséquilibre) consacré à l'étude de la stabilité. Après avoir présenté intuitivement la forme des fonctions de demande (relations prixdemande et prix-valeur de la demande) (Allais, 1993, 386-391) telles qu'elles

27 Cette classification doit servir de point de départ à un exposé des propriétés mathématiques des courbes d'indifférence en fonction des classifications établies (Allais, 1943 et 1993, \$65). Il s'agit en fait de trouver des propriétés analytiques correspondant, en un point donné, aux différents types de couples identifiés. L'objectif analytique est d'établir un coefficient noté $\mathrm{K}_{\mathrm{AB}}$ indiquant le degré de substituabilité (Allais, 1943 et 1993 , \65 et pour une discussion, Chipman (1956) et Charette et Bronsard (1975)). 
résultent des “données de l'observation ", Allais s'engage dans une présentation théorique des lois de la demande (loi externe, loi interne, et loi interne en valeur). A priori, les lois de demande devraient décrire l'ensemble le plus large possible de comportements de demande conformes à l'expérience, sur la base de l'exposé général des courbes d'indifférence. En fait, les choses ne sont pas si simples, car Allais met en avant systématiquement les implications empiriques de l'hypothèse d'indépendance psychologique (ou de faible dépendance psychologique) et justifie ainsi d'en faire une hypothèse centrale pour la suite.

Commençons par présenter les principaux énoncés que Allais tire de l' "observation". Analytiquement, Allais a recours à une expression de la loi de demande à partir de l'équation de Slutsky, qu'il tient sans doute de l'article de Hicks et Allen (1934) ou, plus probablement, de l'opuscule en français de Hicks $(1937)^{28}$. Mais l'originalité de Allais tient plutôt dans son analyse en valeur, qui anticipe sur l'analyse de la stabilité. La valeur de la demande est une fonction croissante puis décroissante du prix. Toute la question est de savoir si le point de retournement de la courbe se situe à un niveau de prix élevé ou faible, dans les termes de Allais. Ainsi, pour les biens de première nécessité (par exemple les biens agricoles) la valeur de la demande croît jusqu'à un niveau élevé du prix. Allais généralise cette situations et considère qu'elle représente bien la plus grande partie des situations concrètes.

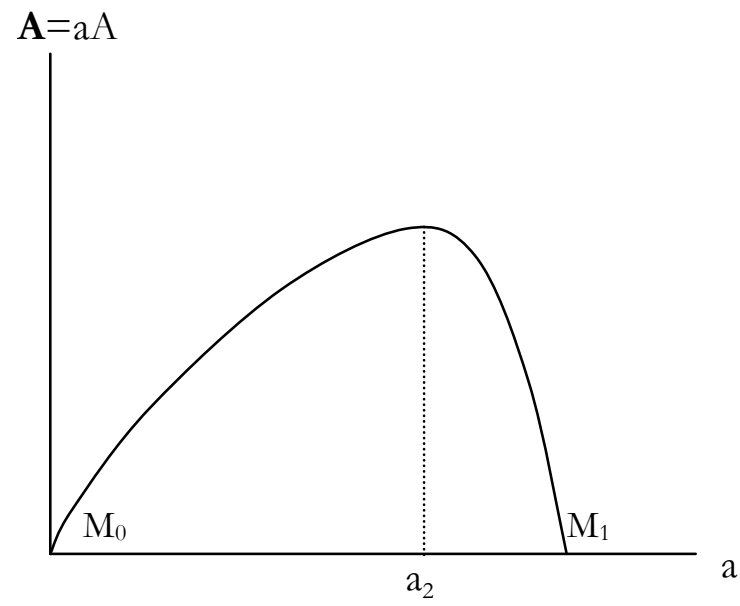

Figure 4

Valeur de la demande. $a$ indique le prix en abscisse, $\mathbf{A}=a \mathrm{~A}$ Source : Allais (1943, $400 ; 1993,390$, figure 2)

De manière générale, dit-il, on peut accepter l'hypothèse que "dans la plus grande partie du domaine de variation du prix, il y a croissance de la valeur de la demande" (Allais, 1943, 401-2, 1993, 391). Par expérience, on se trouverait donc plus souvent dans la configuration de la figure 4, pour laquelle la courbe de demande en valeur est croissante jusqu'à un niveau élevé de prix $\left(a_{2}\right)$

Il est à noter que cette loi de la demande en valeur, qui n'est autre que la loi de Gregory King, n'est plus vérifiée dès que le bien considéré a des substituts ou des complémentaires. Allais $(1943,171 ; 1993,391)$ se débarrasse de cette difficulté en

${ }^{28}$ La distinction entre effets de revenu et de substitution y est exprimée en termes d'élasticité, et c'est à partir de ces termes que Allais analyse les conditions de validité de la loi de la demande. Allais justifie la loi de demande en ayant recours à un principe général de substitution (Allais, 1943, 397; 1993, 387). Les cas exceptionnels de fonctions de demande croissantes se produisent, bien sûr : ils supposent une relation de substituts asymétriques et, comme dans l'exemple du beurre et de la margarine, que le bien inférieur n'ait luimême pas de substitut inférieur, "un fait bien établi par les recherches statistiques" (Allais, 1943, 397 ; 1993 , 387). 
indiquant simplement qu'il faudrait alors considérer la loi de King relativement au groupe de substituts. Ainsi, Allais n'ignore pas les substituts et les complémentaires, il cherche simplement une raison de les négliger en théorie et de se concentrer sur l'hypothèse d'indépendance psychologique. Ce choix découle d'une analyse des comportements de demande et non directement d'une analyse des préférences.

La même question se pose, plus fortement peut-être, lorsqu'on aborde les "lois de variation externe de la demande" (Allais, 1943 et 1993, \$172), c'est-à-dire les relations entre le prix d'un bien et la demande d'autres biens. Ces relations sont essentielles car elles permettront de comprendre comment la variation du prix d'un bien se répercute sur les autres marchés. Pour Allais, l'augmentation du prix du bien A se traduit par une augmentation des autres consommations pour l'offreur, et par une baisse pour le demandeur (si l'on se situe bien sur la portion croissante de la fonction de demande interne en valeur). Implicitement, il se place dans l'hypothèse où les biens sont tous normaux, une hypothèse parfaitement cohérente avec le cas d'une fonction d'utilité additivement séparable ${ }^{29}$. Ce n'est que dans des cas exceptionnels qu'il n'en sera pas ainsi, à chaque fois que deux biens sont dans une relation hiérarchique de substituts asymétriques (Allais, 1943, 407 ; 1993, 396, note 1). Une fois encore, Allais poursuit donc l'idée de négliger les relations trop atypiques de substituabilité qui viendraient troubler les lois de variation externe de la demande.

Venons-en à présent à l'analyse théorique de ces lois de la demande. Allais s'efforce de reproduire analytiquement l'ensemble de ces énoncés, à partir de l'équation de Slutsky, sous l'hypothèse d'indépendance psychologique. Il commence par présenter le cas général, à partir d'une fonction d'utilité générale (Allais, 1993, \$174, et notamment le système d'équations (9)). Ne pouvant en tirer de conclusions précises sur les lois de demande dans ce cas, Allais introduit alors l'hypothèse d'indépendance psychologique, qu'il présente comme suffisamment générale pour donner une bonne approximation des comportements de demande dominants sur les marchés :

“Pour avoir une idée plus précise de ce qui se passe dans le cas général, nous procéderons donc à l'examen du cas particulier de services indépendants, dont la discussion ne présente pas de difficulté. En fait, l'hypothèse de l'indépendance des services ne peut constituer qu'une première approximation de la réalité, mais d'une part et comme nous le verrons on doit la considérer comme la serrant d'assez près et par ailleurs elle permet de retrouver les lois expérimentales de l'offre et de la demande et de fournir très simplement des indications précieuses." (Allais, 1993, 407) ${ }^{30}$

De cette hypothèse d'indépendance, Allais déduit d'abord deux énoncés de statique comparative (Allais, 1993, $\$ 175$, équations 13 et 15) :

1) la demande d'un bien est une fonction décroissante du prix de ce bien.

2) la demande des autres biens varie en sens inverse de la valeur de la demande du bien dont le prix varie.

Ensuite, en s'appuyant à la fois sur un principe de psychologie expérimentale (loi des désirabilités totales décroissante) et sur l'hypothèse d'indépendance psychologique, Allais retrouve bien la loi de variation de la valeur de la demande

${ }^{29}$ Les implications spécifiques d'une fonction d'utilité additivement séparable sur le comportement de demande ont été analysées par Pareto, Slutsky, Hicks et Allen. Allais avait au moins connaissance des propositions de Pareto et de celles de Hicks et Allen. Pour une synthèse historique sur les implications de l'hypothèse d'utilité additive, voir Weber (1999).

$30 \mathrm{Ou}$ encore: “l'hypothèse d'indépendance pour n'être pas rigoureuse n'en constitue pas moins une approximation valable et susceptible de donner une idée de l'allure générale des phénomènes" (Allais, 1993, 407, note 37 ) 
(loi de King) ${ }^{31}$ (Allais, 1993, \$175, équation 23) :

3) En général, la valeur de la demande est une fonction croissante du prix dans la plus grande partie de son domaine de variation.

Enfin, Allais généralise ces énoncés au cas d’une économie walrasienne d'échange pur (Allais, 1993, 421), telle que le revenu des échangistes est donné par la valeur de leurs dotations initiales $\left(\mathbf{A}_{0}, \mathbf{B}_{0}, \ldots\right)$ (Allais, 1993, \$176, équations 1, 2 et 3) :

4) La demande d'un bien varie en sens inverse de son prix

5) La valeur de l'offre d'un bien varie dans le même sens que son prix

6) La valeur de la demande [resp. de l'offre] des autres biens varie dans le même sens que la valeur de l'offre [resp. de la demande] du bien dont le prix varie.

En définitive, les énoncés 4), 5) et 6) sont les énoncés de base sur les comportements à partir desquels Allais va se lancer dans l'analyse de la stabilité, énoncés qu'il devra compléter d'une manière ou d'une autre pour pouvoir dire quelque chose sur le sens de variation des valeurs des demande excédentaires (internes et externes). On retiendra simplement que tous les énoncés qui précèdent sont en conformité avec les comportements induits par l'hypothèse d'indépendance psychologique, et par son corollaire, une fonction d'utilité additivement séparable.

\subsection{La stabilité de l'économie walrasienne}

Lorsqu'il publie son Traité, Allais a raison d'affirmer que le problème de la stabilité de l'équilibre "n'a jamais fait l'objet d'une étude générale et systématique qui puisse être considérée comme satisfaisante" (Allais, 1943, 271 ; 1993, 268). Par rapport aux remarques faites par Hicks en 1937, l'analyse de Allais présente une ressemblance et une différence importantes. Comme chez Hicks, mais aussi comme chez Walras, l'étude de la stabilité consiste à généraliser la loi de l'offre et de la demande (Allais, 1943, 270, 1993, 267). Seulement, Allais voit bien que le mécanisme de l'offre et de la demande sur un marché particulier n'est pas suffisant pour permettre une description théorique rigoureuse de la stabilité d'une économie en déséquilibre, et qu'il est indispensable de tenir compte du fait que "dans la réalité tous les marchés évoluent simultanément" (Allais, 1943, 484 ; 1993, 470). Une véritable analyse de la stabilité d'un système économique doit donc mettre en évidence les propriétés des réactions simultanées des différents marchés dans leurs interdépendances : “il reste à démontrer que l'évolution de chaque marché élémentaire est telle que l'ensemble des marchés évolue vers l'équilibre" (Allais, 1943, 486 ; 1993, 471).

Avant de rentrer dans le détail du raisonnement de Allais, on peut faire deux remarques.

1) L'originalité de Allais est de produire une démonstration de la stabilité à partir d'un raisonnement sur les valeurs des demandes excédentaires, alors que Walras ou Hicks raisonnent sur les demandes excédentaires ${ }^{32}$. Toutes les lois de

31 “Ainsi la considération des résultats obtenus en psychologie expérimentale permet de retrouver théoriquement et dans le cas de biens indépendants la loi expérimentale de Gregory King. ” (Allais, 1993, 413)

32 Une comparaison systématique de Hicks et Allais montrerait plus largement que Hicks ne cherche pas à proposer une démonstration de la stabilité d'un tâtonnement, mais simplement à savoir si cet ajustement est rapide ou lent (selon que la stabilité est parfaite ou imparfaite). Cette idée conduit Hicks à formuler des conditions de stabilité qui s'apparentent formellement avec des conditions de second ordre de maximisation individuelle des agents. Pour autant, chez Hicks comme chez Allais, la stabilité de l'équilibre résulte de l'action de la loi de l'offre et de la demande et des comportements individuels des agents (Hicks, 1939, 66) 
comportement établies précédemment (4), 5) et 6)) trouveront ainsi leur utilité ${ }^{33}$. 2) Tels quels, les énoncés précédents ne suffisent pas à mener l'analyse des interactions entre les marchés. Il faudra dans un premier temps que Allais dise quelque chose sur le sens de variation de la demande excédentaire d'un bien avec son prix. Il faudra ensuite qu'il dise quelque chose sur le sens de variation de la demande excédentaire des autres biens.

Le principe de la démonstration consiste à établir la décroissance d'une "fonction caractéristique", qui est en fait la valeur absolue des fonctions de demande excédentaire en valeur ${ }^{34}$ :

Si l'on considère la somme

$$
\mathbf{H}=\sum_{B}\left|\mathbf{B}^{S}-\mathbf{B}^{D}\right|
$$

des valeurs absolues des différences entre les valeurs des offres $\left[\mathbf{B}^{\mathrm{S}}\right]$ et celles des demandes $\left[\mathrm{B}^{\mathrm{D}}\right]$, il est possible de montrer, sous certaines conditions vraisemblables, que cette somme que nous appellerons "fonction caractéristique", ne peut que décroître, lorsqu'un marché élémentaire quelconque évolue conformément à la loi d'évolution des prix. Il en résulte que d'une part l'ensemble de l'Économie considérée évolue vers une position d'équilibre bien déterminée définie par l'égalité des offres et des demandes et par ailleurs que cette position d'équilibre est stable. (Allais, 1943, 486-487; 1993, 472, nos italiques)

La démonstration repose sur l'analyse des variations des valeurs des demandes excédentaires, en distinguant le comportement des offreurs et celui des demandeurs. Allais mène l'analyse à partir d'un marché $A$ en déséquilibre, choisi arbitrairement, tel que l'offre en valeur excède la demande en valeur $\left(\mathbf{A}^{\mathrm{S}}>\mathbf{A}^{\mathrm{D}}\right)$. Allais sépare les autres marchés en deux groupes, selon qu'ils sont en excès de demande ou en excès d'offre. Il faut alors montrer que la valeur absolue de la somme des valeurs des demandes excédentaires sur tous les marchés pris ensemble diminue tant que l'équilibre n'est pas réalisé sur tous les marchés. Le cœur de la démonstration réside donc dans l'établissement des conditions qui garantissent que les variations successives des prix sur le marché $A$, conformément au principe de la loi de l'offre et de la demande, ont pour effet de réduire (et non d'augmenter) $H^{35}$.

Pour conduire cette démonstration, il faut partir des lois de l'offre et de la demande d'une économie walrasienne d'échange où les biens sont indépendants (Allais, 1943, 432 ; 1993, 421). Ces "lois du marché walrasien" généralisent simplement les lois de l'offre et de la demande à une économie d'échange, le revenu étant alors déterminé par la valeur des dotations individuelles (Allais, 1943, 432 ; 1993, 421). La démonstration suppose donc de faire, dans un premier temps, des hypothèses sur le comportement de demande et d'offre sur le marché $A$, puis, à partir de ces hypothèses, de discuter l'effet des variations du prix $p_{a}$ sur les autres marchés.

L'analyse de l'offre, dans le Traité, est moins détaillée que celle de la demande. On en retiendra simplement que la valeur de l'offre, même dans une économie

\footnotetext{
33 L'avantage d'un raisonnement à partir des valeurs des demandes excédentaires tient, selon Allais, au fait que les courbes de demande et d'offre en valeur possèdent une "permanence" (Allais, 1943, 481; 1993, 466) qui est favorable à la généralité du résultat. En fait, il faut bien voir que les lois de demande en valeur utilisées par Allais supposent au départ certaines relations entre prix et quantités, et que la discussion de l'évolution de la fonction caractéristique s'en trouve simplifiée.

34 Nous avons légèrement modifié la notation originale de Allais. Notamment, les symboles de valeurs sont représentés en caractère gras, tandis que les symboles de quantités sont en caractère maigre. Ainsi, $\mathbf{B}=$ $\mathrm{p}_{\mathrm{b}} B$.

35 Pour une autre formulation, voir Weintraub (1991b, 390)
} 
d'échange, est une fonction croissante du prix (Allais, 1993, 387). Dans l'ensemble, Allais raisonne à la manière de Walras, avec l'idée que les comportements d'offre sont contraints par les comportements de demande et s'en déduisent logiquement. Cette idée se retrouve notamment dans l'usage de la loi de Walras pour établir la décroissance de la fonction caractéristique. Allais va alors préciser le sens de variation des valeurs des demande excédentaires. L'objectif de Allais est de garantir au départ que les valeurs des demandes excédentaires de chaque service sont bien des fonctions décroissantes du prix de ces services. Si la valeur de la demande de $A$ varie en sens inverse de son prix, la valeur de la demande excédentaire est biens décroissante. Mais la situation la plus courante, on l'a vu, est que la valeur de la demande varie dans le même sens que le prix. Dans cette situation, il n'est pas exclu que la fonction d'offre en valeur coupe la fonction de demande en valeur par le haut. Pour éviter cette situation, Allais introduit un premier lemme. Ce premier lemme concerne l'effet de la variation $\mathrm{d} p_{a}$ sur la valeur de la demande excédentaire $\operatorname{de} A$.

Lemme 1 : Variation avec le prix de l'excès de la valeur de la demande.

Sous l'hypothèse que "les différents individus ont des psychologies et des ressources pas trop différentes", leur comportement de consommation sera relativement homogène, et alors, "au voisinage de l'équilibre, et pour des prix croissants, la valeur de l'offre croît plus vite que celle de la demande" (Allais, 1943, 488).

Autrement dit, dans tous les cas, la valeur de la demande excédentaire d'un bien décroît avec son propre prix ${ }^{36}$. On sera donc dans l'une des configurations de la figure 5 ( $\mathrm{a}$ et $\mathrm{b})$ :

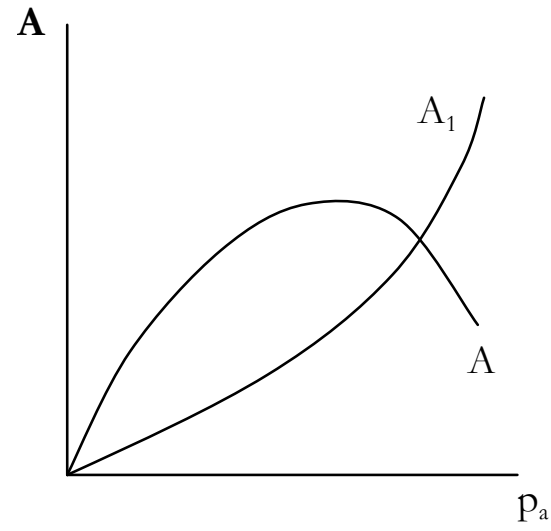

Figure 5(a)

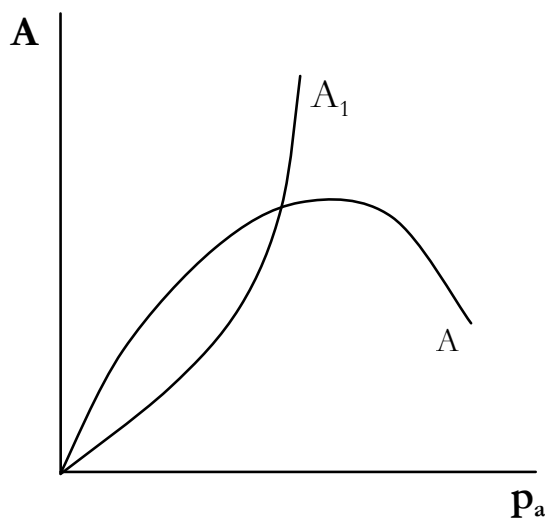

Figure 5(b)

\section{Deux configurations possibles de la demande excédentaire (A- $\left.A_{1}\right)$} au voisinage de l'équilibre.

Source : Allais (1943, 487 ; 1993, 473, fig.1 et 2)

Ce lemme 1 permet donc de garantir que la loi de l'offre et de la demande conduit à un équilibre sur chaque marché. Un second lemme permet alors d'envisager les implications de la variation du prix $\mathrm{d} p_{a}$ sur les autres marchés. Ce second lemme s'applique explicitement au cas le plus général de la figure 5(b) mais le raisonnement peut s'étendre aisément aux situations exceptionnelles de la figure $5(\mathrm{a})$.

Lemme 2: Répercussion sur les autres marchés.

Considérons n’importe quel autre marché, représenté de manière générique par le

36 Allais raisonne en termes d'offre excédentaire. Il montre donc que l'offre excédentaire, en valeur, est croissante. 
marché du bien B. Dans le cas le plus fréquent de la figure 5(b), au voisinage de l'équilibre, si $\mathrm{d} p_{a}<0$, alors :

$$
0<-\delta \mathbf{A}^{D}<-\delta \mathbf{A}^{S}
$$

Pour exposer clairement la répercussion sur le marché $B$, il faut introduire une distinction entre les offreurs et les demandeurs de $A$ qui interviennent sur ce marché.

On indice par $i$ les offreurs de $A$, en distinguant deux sous-groupes :

$i$ les offreurs de $A$ demandeurs de $B$

$i$ "les offreurs de $A$ offreurs de $B$

On indice par $j$ les demandeurs de $A$, en distinguant deux sous-groupes :

$j$ 'les demandeurs de $A$ demandeurs de $B$

$j$ "les demandeurs de $A$ offreurs de $B$

Le lemme 2 exprime que la répercussion sur le marché $B$ vérifie l'inégalité suivante :

$$
0<\sum_{j^{\prime}} \delta \mathbf{B}_{j^{\prime}}^{D}-\sum_{j^{\prime \prime}} \delta \mathbf{B}_{j^{\prime \prime}}^{S}<\sum_{i^{\prime \prime}} \delta \mathbf{B}_{i^{\prime \prime}}^{S}-\sum_{i^{\prime}} \delta \mathbf{B}_{i^{\prime}}^{D}
$$

Cette inégalité signifie

que l'augmentation de la valeur algébrique de la demande du service $(B)$ de la part des demandeurs [] du service $(A)$ est inférieure à l'augmentation de la valeur algébrique de l'offre du service $(B)$ de la part des offreurs [i] du service (A). (Allais, 1943, 489)

On aura remarqué que ces deux lemmes sont sujets à des hypothèses nouvelles sur les préférences et les comportements individuels, que nous commenterons par la suite. Muni de ces deux lemmes, Allais va établir la stabilité d'une économie walrasienne.

Allais suppose un marché élémentaire $A$ en déséquilibre (offre excédentaire) et étudie les répercussions, sur la fonction caractéristique, de ce déséquilibre. Il commence par distinguer les biens pour lesquels on observe un excès d'offre en valeur $\left(\mathbf{B}^{\mathrm{S}}>\mathbf{B}^{\mathrm{D}}\right)$ de ceux pour lesquels on observe un excès de demande en valeur $\left(\mathbf{C}^{\mathrm{D}}>\mathbf{C}^{\mathrm{S}}\right)$. Donc, la fonction caractéristique devient :

$$
\mathbf{H}=\mathbf{A}^{S}-\mathbf{A}^{D}+\sum_{B}\left(\mathbf{B}^{S}-\mathbf{B}^{D}\right)+\sum_{C}\left(\mathbf{C}^{D}-\mathbf{C}^{S}\right)
$$

D'après la loi de Walras, la valeur totale des excès d'offre est égale à la valeur totale des excès de demande. La fonction caractéristique peut donc s'écrire :

$$
\mathbf{H}=2 \sum_{C}\left(\mathbf{C}^{D}-\mathbf{C}^{S}\right)
$$

Il s'agit alors d'apprécier l'effet de la variation du prix de $A\left(\mathrm{~d} p_{\mathrm{a}}<0\right)$ consécutive au déséquilibre sur le marché de ce bien $\left(p_{\mathrm{a}} \mathrm{A}^{\mathrm{s}}>p_{\mathrm{a}} \mathrm{A}^{\mathrm{d}}\right.$, c'est-à-dire $\left.\mathbf{A}^{\mathrm{s}}>\mathbf{A}^{\mathrm{D}}\right)$ sur la variation de la valeur de la fonction caractéristique $H$.

Deux cas sont alors étudiés. Le cas où la valeur de la demande de $A$ varie pour chaque individu dans le sens inverse du prix et le cas où elle varie dans le même sens que le prix. Dans le second cas, le lemme 1 est indispensable pour exclure des situations qui invalideraient la loi de l'offre et de la demande en valeur et le lemme 2 permet de garantir (dans tous les cas) que les répercussions sur les autres marchés vont dans le sens d'une réduction de la valeur de la demande

37 Le même raisonnement appliqué à la situation de départ 5(a) : $-\delta \mathbf{A}^{D}<0<-\delta \mathbf{A}^{S}$ donnerait un résultat identique. 
excédentaire ${ }^{38}$. Cela permet à Allais $(1943,490-1$; 1993, 476-7) de préciser le sens de variation de l'équation caractéristique. Ce raisonnement est valable quel que soit le marché élémentaire sur lequel on fait l'hypothèse initiale d'un déséquilibre en valeur:

Ainsi on voit que dans tous les cas l'évolution d'un marché particulier entraîne une diminution de la somme des valeurs absolues des différences entre les valeurs des demandes et celles des offres. Comme ce mécanisme joue tant que chacune de ces différences n'est pas nulle, on voit que sous l'action du mécanisme économique le système évolue vers un état où pour tout service la valeur de l'offre est égale à celle de la demande. (Allais, 1943, $491 ; 1993,477)$

La stabilité est donc finalement établie, sous un ensemble de conditions dispersées dont Allais semble persuadé qu'elles sont acceptables, par conformité avec l'expérience quotidienne ou l'introspection psychologique. Si le projet de Allais est donc bien de montrer que la stabilité est conforme à l'expérience et qu'elle est très vraisemblable, il est indispensable de revenir sur l'ensemble de ces conditions, c'est-à-dire sur l'ensemble d'hypothèses semées au cours du voyage. Nous serons mieux en mesure d'évaluer si Allais parvient à rester étroitement fidèle aux principes généraux de méthode dont il se réclame.

\section{Du BON USAGE DE LA PSYCHOLOGIE DANS LE TRAITE}

Allais est parfaitement conscient que sa démonstration repose finalement sur un ensemble d'hypothèses qui, "pour être vraisemblables, n'en sont pas moins restrictives" (Allais, 1943, 486 ; 1993, 472). Au moment de résumer l'ensemble d'hypothèses qui donnent corps à sa démonstration de la stabilité d'une économie walrasienne, il distingue deux ensembles d'hypothèses suffisantes, les unes de nature psychologique, les autres liées à la distribution des caractéristiques individuelles dans la population (Allais, 1943, 494 ; 1993, 480) ${ }^{39}$ :

1/ la faible dépendance des désirabilités (formellement l'indépendance psychologique) et la décroissance des désirabilités.

2/ l'existence d'un degré modéré de hasard dans la distribution des caractéristiques psychologiques des individus et de leurs revenus.

Afin de préciser la place des fondements psychologiques de la théorie de l'équilibre général chez Allais, on procédera en deux temps. Dans un premier temps, il est indispensable de démêler, autant que possible, le rôle des différentes hypothèses dans la démonstration. On verra à cette occasion que l'hypothèse d'indépendance psychologique ne joue pas un rôle direct dans la démonstration. (3.1. L'homme moyen, l'indépendance psychologique et la stabilité). Dans un second temps, on s'interrogera plus largement sur la place de l'hypothèse d'indépendance psychologique, dans ses usages tant rhétoriques, heuristiques et analytiques. On montrera ainsi que Allais instrumente d'autant plus cette hypothèse, nous invitant ainsi à reconsidérer la logique méthodologique à l'œuvre derrière l'argumentation analytique (3.2. Retour sur l'hypothèse d'indépendance psychologique dans le Traité).

\footnotetext{
38 Les évolutions simultanées (et symétriques) de tous les marchés se renforcent. C'est une différence importante avec Hicks. Dans Valeur et capital, en effet, la distinction entre stabilité parfaite et imparfaite autorise que certaines interdépendances entre marchés ne rapprochent pas de la solution d'équilibre général.

39 Plus précisément, Allais propose une liste d’hypothèses censées généraliser les conditions suffisantes de la stabilité de l'économie d'échange au cas d'une économie de production. Nous nous contentons d'énumérer ici les hypothèses relatives au comportement de demande.
} 


\subsection{L'homme moyen, l'indépendance psychologique et la stabilité}

On peut partir d'un constat en forme d'énigme : si on se limite strictement au raisonnement qui structure la démonstration de la stabilité d'un équilibre général walrasien, on remarque que Allais s'appuie exclusivement sur les lemmes 1 et 2 et qu'il introduit à cette occasion une hypothèse sur la concentration des préférences et des dotations individuelles autour d'un profil moyen. En conséquence, une première lecture rapide laisse penser que ces deux lemmes sont construits indépendamment de l'hypothèse d'indépendance psychologique et d'une fonction d'utilité additivement séparable. Comment expliquer alors que Allais attribue une telle importance aux hypothèses psychologiques dans le Traité? On pourrait choisir d'ignorer la difficulté, mais on cherchera plutôt ici à reconstruire l'attachement pour cette hypothèse et la fonction analytique que Allais lui prête, en dernier ressort.

Plusieurs possibilités s'offrent à nous. On pourrait interpréter ce moment comme la marque d'une inattention, ou comme la volonté d'entretenir l'illusion selon laquelle cette hypothèse serait impliquée de près ou de loin dans la démonstration, feinte rhétorique destinée à augmenter artificiellement l'attrait et le réalisme de la démonstration. Mais avant d'en venir à de telles explications, il faut examiner de plus près la fonction strictement analytique de l'hypothèse d'indépendance psychologique et ne pas conclure précipitamment à son inutilité analytique. Le travail d'interprétation nécessaire suppose néanmoins de multiplier les niveaux de lecture. Il faut démêler autant que possible le rôle des différentes hypothèses dans la démonstration, c'est-à-dire dans l'établissement des lemmes 1 et 2 .

D'un point de vue strictement analytique, on doit remarquer trois choses : Premièrement, la "donnée d'observation" qu'est la loi de demande interne en valeur (ou loi de King) n'est établie que sous l'hypothèse conjointe d'indépendance psychologique et de désirabilité absolue. L'hypothèse d'indépendance psychologique est donc motivée ici par la nécessité d'établir la stabilité dans le cas le plus général, c'est-à-dire pour Allais dans un cas empiriquement significatif, d'une fonction de demande en valeur croissante des prix.

Deuxièmement, l'hypothèse d'indépendance psychologique permet d'obtenir des énoncés bien précis sur les lois de demande externe en valeur $\left(\partial \mathbf{B} / \partial p_{a}\right)$, ce qui n'aurait pas été le cas avec une fonction d'utilité différente.

Troisièmement, le lemme 1 est démontré sous l'hypothèse d'indépendance psychologique. En effet, il ne dispose pas, dans le cas général, d'une relation entre le prix d'un bien et la valeur de ce bien (offerte ou demandée).

Finalement, on voit se dessiner un usage répétitif de l'hypothèse, qui permet de comprendre que Allais ne cherche pas à examiner dans le détail de chaque énoncé la nécessité analytique de l'hypothèse d'indépendance psychologique.

En fait, il nous semble plus exact de considérer que les hypothèses introduites à l'occasion des lemmes 1 et 2 sont des hypothèses complémentaires des hypothèses psychologiques et que de ce fait Allais envisage ces hypothèses comme un tout, sans chercher à relever le mérite de telle ou telle hypothèse. À cet égard, l'intérêt des lois de demande externe en valeur est d'indiquer clairement quel type d'argument supplémentaire est nécessaire pour construire une démonstration de la stabilité. Il y a donc un enjeu réel pour la démonstration de la stabilité, dans le sens où l'hypothèse d'indépendance psychologique permet à Allais de mesurer les besoins exacts de propriétés nouvelles, sans doute moins réalistes mais non moins 
nécessaires du point de vue de la rigueur du raisonnement. Notamment, on peut se souvenir que l'hypothèse d'indépendance psychologique garantit que les effets de revenus seront faibles. Cette propriété autorise donc d'autant plus à considérer que les hypothèses nouvelles complémentaires pourront n'être vérifiées que statistiquement, et qu'une dose non négligeable de dispersion des préférences ("un degré modéré de hasard") ou des dotations ne remettrait pas en cause l'argument. Un tel raisonnement n'aurait pas été possible sans l'hypothèse d'indépendance psychologique, car l'existence d'effets de revenus plus importants l'aurait rendu plus fragile.

Ainsi, comme l'a dit Negishi (1962), il y a bien dans le Traité un ensemble d'hypothèses comparables à la substituabilité brute. En effet, le résultat final est obtenu par sommation de la propriété selon laquelle sur chaque marché B autre que le marché élémentaire $\mathrm{A}$, on a

$$
\frac{\partial\left(p_{b} B^{D}-p_{b} B^{S}\right)}{\partial p_{a}}>0
$$

Cette propriété revient à supposer que sur tous les autres marchés que $\mathrm{A}$,

$$
\frac{\partial\left(B^{D}-B^{S}\right)}{\partial p_{a}}>0,
$$

ce qui correspond bien à l'hypothèse de substituabilité brute. Mais remarquons immédiatement que Allais n'emploie jamais cette définition et qu'il justifie d'une manière toute différente sa "démonstration". Dans la mesure où Allais relie sa démonstration à l'hypothèse d'indépendance psychologique, il ne pouvait mettre en avant le rôle de la substituabilité brute dans le système, sauf à brouiller le sens de sa construction. À la différence d'une analyse uniquement en termes de fonctions de demande excédentaire, caractéristique des recherches des années 1950 et 1960, et principalement héritée de Hicks, sa construction tient en un mélange de comportements de demande et d'hypothèses psychologiques. Par contraste, elle fait ressortir les hypothèses introduites par Allais relatives aux caractéristiques des agents, les plus éloignées de la méthodologie générale du Traité.

L'idée, c'est que pour pouvoir produire le lemme 1 et 2 , surtout 2, l'hypothèse d'indépendance psychologique élimine les objections possibles sur l'ampleur des effets de revenu. Elle n’y suffit pas à elle seule mais elle tend à la justifier. Allais n'explicite jamais comment quelle complémentarité il voit dans ses différentes hypothèses, et nous devons nous contenter d'une reconstruction hypothétique.

Les hypothèses sur les caractéristiques des agents sont introduites pour établir les lemmes 1 et 2 . Allais s'appuie alors sur l'idée que les réactions des agents sont à peu près les mêmes, qu'ils soient offreurs ou demandeurs. Le meilleur moyen d'y parvenir est de prendre pour référence une population faite d'individus identiques dans leurs goûts et leurs dotations. Pour cela, Allais suppose que "les différents individus ont des psychologies et de ressources pas trop différentes". Alors, "sur un marché walrasien dont les individus présentent une certaine uniformité, tout point d'équilibre, c'est-à-dire tout point où l'offre et la demande sont égales, est un point d'équilibre stable" (Allais, 1943, 488 ; 1993, 474, note 7).

En fait mathématiquement, Allais raisonne à partir du cas extrême dans lequel les individus sont identiques. Selon lui, la démonstration rigoureuse pourrait être établie

(...) en considérant les distributions statistiques des revenus et des fonctions de satisfaction. On verrait que la propriété indiquée suppose essentiellement que la grande masse des individus est concentrée autour d'un type moyen, et que si cette condition est remplie, elle reste encore vraie, même si certains 
individus s'en écartent notablement. Cette propriété résulte donc d'un degré modéré de hasard dans la répartition des individus suivant leurs goûts et leurs ressources. (Allais, 1943, 488, 1993, 474, note 8) 40

En fait Allais est, semble-t-il, assez convaincu par les conséquences auxquelles conduit cette hypothèse, notamment pour le lemme 2 :

Il y a là une propriété [le lemme 2] que l'on peut considérer comme intuitive. Si en effet sur un marché donné la répercussion de la variation du prix a une incidence plus faible sur les valeurs des demandes que sur celles des offres, cela signifie que, d'une manière générale, l'incidence de la variation du prix considérée est plus faible sur les déterminations des demandeurs que sur celles des offreurs. (Allais, 1943, 489 ; 1993, 475-6)

On peut aller plus loin et tenter une justification du lemme 2 à partir des propriétés supposées de l'indépendance psychologique. Parmi les quatre catégories d'agents identifiées par Allais pour justifier le lemme 2, on peut remarquer qu'il y en a qui sont exclusivement offreurs sur les deux marchés, d'autres qui sont exclusivement demandeurs, d'autres enfin qui sont offreur sur l'un et demandeur sur l'autre. Ces derniers jouent un rôle symétrique, dans l'esprit de Allais, et leur comportement tend à s'annuler. Ne reste plus qu'à comparer les réactions de ceux qui sont exclusivement demandeurs et de ceux qui sont exclusivement offreurs. En raison de l'hypothèse d'indépendance psychologique, Allais considère que les demandeurs réagiront peu car ils répercuteront une variation de prix sur l'ensemble de tous les biens qu'ils demandent, tandis que du point de vue des offreurs, les effets sur les autres marchés seront plus élevées car les revenus proviennent essentiellement de l'offre d'un petit nombre de biens (Allais, 1993, 422, note 3) ${ }^{41}$.

Dans tous les cas, cette justification relève davantage d'une démarche heuristique et intuitive que d'une construction analytique solide, et il va de soi qu'elle revient à prêter à l'hypothèse d'indépendance psychologique des implications empiriques qu'elle ne possède pas. Elle désigne en revanche l'hypothèse d'indépendance psychologique comme une hypothèse récurrente qui structure toute la logique de la démonstration et de la justification dans le Traité.

\subsection{Retour sur l'hypothèse d'indépendance psychologique}

Pour Allais, donner des conditions suffisantes de stabilité d'un équilibre général ne constitue un objectif digne d'intérêt que dans la mesure où ces conditions sont vraisemblables et correspondent à une approximation raisonnable des comportements individuels qui concourent à cette stabilité. Le résultat ne devrait pas être obtenu au détriment de fondements microéconomiques conformes aux données de l'introspection. C'est pourquoi l'analyse des préférences et des choix occupe une place si importante dans le Traité. Elles sont au cœur de la validation des énoncés de la théorie de la demande et de toute la mécanique du Traité. Commentant la théorie du choix élaborée en 1943, Allais écrira d'ailleurs :

\footnotetext{
40 On remarquera que Allais reproduit presque à l'identique une expression utilisée par Hicks (1937, 29) pour justifier la stabilité : "Il y a un degré modéré de hasard dans la distribution des goûts et des revenus entre les acheteurs et vendeurs d'un bien déterminé, de telle sorte que le terme du revenu est modérément faible".

${ }^{41}$ En effet, on peut se demander si le caractère "intuitif" de ce résultat ne vient pas, pour Allais, du fait que les offreurs tirent généralement leur revenu de la vente de deux ou trois biens et services, tout au plus (Allais, 1993, 422, note 3). Cela justifierait leur grande sensibilité à la variation du prix de l'un des biens qu'ils offrent, tandis que les demandeurs seraient moins sensibles puisqu'ils répartissent leur dépense sur de nombreux biens. Si cela paraît séduisant, il n'est pas pour autant certain que cela soit compatible avec le fait que les agents doivent avoir des ressources semblables, selon Allais. Cela n'aurait pas de sens dans le cadre d'une économie d'échange, à moins de comprendre cette hypothèse comme portant sur les valeurs des ressources. Voir aussi Weintraub (1991b, 392).
} 
L'analyse que nous avons donnée a l'avantage de montrer, avec une parfaite clarté, que la théorie générale des surfaces d'indifférence, des lois d'offre et de demande, et de la stabilité constitue un tout parfaitement cohérent dont les différentes parties se confirment mutuellement et qu'elle acquiert par là même un très haut degré de certitude. (Allais, 1993, Introduction à la deuxième édition, 24)

L'hypothèse d'indépendance psychologique est donc essentielle, car c'est par cette hypothèse que Allais assure l'ancrage réaliste de son modèle d'équilibre général. Il n'est donc pas étonnant de constater combien cette hypothèse est l'objet de toutes les attentions, patiemment construite et présente à toute les étapes de l'analyse. Pour autant, sa validation a posteriori dans le Traité relève d'une interprétation assez libérale des intentions méthodologiques de Allais. En principe, nous devrions distinguer deux choses: la justification de l'hypothèse, d'une part, par généralisation d'arguments introspectifs; les implications de cette hypothèse sur les comportements de marché, d'autre part, considérées comme autant de confirmations de sa validité. La difficulté tient justement au fait que Allais n'hésite pas à procéder par allers et retours entre les arguments introspectifs et d'expérience. Notre objectif, ici, sera de démêler les arguments de Allais et de faire ressortir la manière dont il met en œuvre les principes méthodologiques affichés au début du Traité.

Il y a d'abord une raison analytique essentielle pour que Allais ne puisse fonder l'hypothèse d'indépendance psychologique (ou d'une fonction d'utilité additivement séparable) sur une base simplement introspective. Allais sait bien (par Hicks et Allen (1934) ou par Hicks (1937)) que les propriétés introspectives des préférences n'ont que peu d'implications géométriques (et donc aussi en termes de choix) et qu'inversement les comportements de demande peuvent rarement être associés à des propriétés introspectives des préférences ${ }^{42}$. Allais remarque ainsi que toute la typologie géométrique des biens substituts, indépendants et complémentaires n'est associée qu'à des qualités introspectives "vraisemblables" et que cette typologie, finalement, "se trouvera justifiée par la cohérence des déductions auxquelles elle permettra d'arriver" (Allais, 1943, 131 ; 1993, 133, note 6) ${ }^{43}$. Il opère cependant un rapprochement entre les deux à l'occasion d'un long développement sur l'utilité cardinale (satisfaction absolue) (Allais, 1993, 157-177), en supposant notamment que les biens substituts sont en aussi grand nombre que les biens compléments (Allais, 1993, 168, note 21 ; 169, note 25 ; 174). Par cette hypothèse nouvelle et non discutée, Allais se donne ainsi la possibilité de raisonner indifféremment à partir de propriétés supposées des fonctions d'utilité ou de propriétés, validées par l'expérience, des comportements de marché.

De plus, pour ne retenir de sa typologie que la situation d'indépendance

42 On ne peut inférer précisément les connaissances de Allais sur la question. L'article de Hicks et Allen (1934) ou l'opuscule de Hicks (1937) sont simplement mentionnés. Allais a-t-il par exemple à l'esprit les développements que Hicks et Allen consacrent à l'hypothèse d'une fonction additivement séparable? A t-il pu avoir connaissance de l'article de Slutsky (cité par Hicks (1937) et notamment de la section 10 de cet article consacré aux implications d'une fonction additivement séparable ? A t-il en tête les développements de Fisher (1891) qui fait référence à la définition de la substituabilité à partir de la dérivée seconde croisée de la fonction d'utilité (définition Auspitz-Lieben-Edgeworth-Pareto) ? Pour un parcours historique des contributions à l'analyse des fonctions d'utilité additivement séparables, voir Weber. Pour une discussion des implications empiriques de l'hypothèse de la substituabilité au sens de Auspitz-Lieben-Edgeworth-Pareto, voir Chipman (1977) et Weber (2000).

43 On peut faire à cette définition le même reproche que celui qu'adressait Schultz (1938, 612 et 619) aux définitions d'Allen et de Friedman. De telles définitions nous obligent à connaitre le comportement des consommateurs aux frontières de la satiété, car c'est par les propriétés des lignes de satiété que se fait la classification. 
psychologique, Allais se doit de justifier d'ignorer les relations exceptionnelles de substituts et complémentaires accusés. Pour cela, Allais n’introduit pas de nouveaux arguments psychologiques, mais des propositions relatives aux comportements de demande pour ces biens. Il suppose en effet qu'il est possible d'isoler en un groupe homogène les biens fortement substituables ou fortement complémentaires. La possibilité de faire de tels regroupements est très douteuse, surtout si l'on souhaite simultanément prendre en compte les substituts et les complémentaires. Allais s'appuie en fait sur certaines données "d'expérience" relatives à la sensibilité de la demande. Pour les biens substituts, la valeur totale de la demande de deux biens substituts serait peu sensible aux variations du prix de l'un de ces deux biens. Il suggère donc de considérer les "différents groupes de service à forte substituabilité comme formant des services abstraits" (Allais, 1943, 442 ; 1993, 431), qui se comporteront dès lors "comme des services normaux à faible substituabilité". De cette manière les effets de revenus sont confinés à l'intérieur du groupe de ces biens : 'Les phénomènes de substitution n'ont alors et en première approximation d'autre répercussion que des modifications de répartition du revenu à l'intérieur de chaque groupe" (Allais, 1943, 442 ; 1993, 431). Identiquement, pour les biens fortement complémentaires, le rapport des quantités consommées de ces biens est approximativement fixe, si bien que l'on pourrait les regrouper dans un service unique, ce qui permettrait de négliger également les phénomènes de complémentarité accusée dans le système économique (Allais, 1943, 442 ; 1993, 431). C'est un premier exemple d'application des positions méthodologiques de Allais sur les liens entre l'introspection et les "phénomènes observés" (considérés en fait comme des propriétés banales, voire évidentes). Nous sommes là bien sûr en présence de ces mouvements d'aller et retour entre les données de l'introspection (ou de l'intuition) et les énoncés observables. Cette justification pourra bien paraître parfaitement ad hoc dans sa manière de mêler inextricablement les données introspectives à celles de l' "expérience". Elle n'en est pas moins ingénieuse, et certainement plus élaborée que ce que l'on peut trouver au même moment dans Valeur et Capital. Cela tient, bien sûr, au choix de méthode affiché au commencement.

Plus généralement, le lecteur du Traité peut constater que la progression de l'ouvrage obéit à un rituel immuable. Sur chaque question théorique, Allais donne dans un premier temps un exposé intuitif, puis dans un second temps il en donne un exposé analytique dont la conformité avec les données de l'expérience (statistique ou introspective) est interprétée comme une confirmation de celles-ci. La situation est particulière, toutefois, pour ce qui concerne les préférences, dont les hypothèses de départ "raisonnables" doivent valider les comportements de demande théoriques, tandis que simultanément, la conformité de ces comportements de demande avec l' "expérience" viendrait justifier les restrictions générales faites sur les préférences. La justification de l'hypothèse d'indépendance psychologique dans le Traité et l'utilisation subséquente de propriétés cardinales (satisfactions absolues) suppose donc une interprétation plus instrumentaliste de la théorie des choix qu'il n'y paraît. Ainsi, le but avoué de Allais est d'établir la fécondité de cette hypothèse pour l'analyse du choix et de l'équilibre, en rendant possible une "explication simple des phénomènes observés" (Allais, 1943, 144 ; 1993,146-7).

On a vu en section 2 toutes les implications analytiques de l'hypothèse d'indépendance psychologique et comment Allais construisait cette hypothèse à partir d'une donnée empirique qu'il généralise : la loi de demande interne en valeur. Cet énoncé est en fait celui qui permet à Allais d'enclencher le raisonnement sur la stabilité à partir d'une fonction d'utilité additivement séparable. On ne cachera pas 
les difficultés d'interprétation que suscite cette construction, qui renverse complètement la logique de la justification dans le Traité, de telle manière que l'hypothèse d'indépendance psychologique est utilisée à seule fin de rationaliser en fait certaines données d'observations présentées comme générales. La forme de la demande en valeur est justifiée intuitivement comme résultant d'une donnée d'observation, sorte de généralisation d'une observation statistique pour des biens de première nécessité. Généraliser cette relations à l'ensemble des biens, cela revient en quelque sorte à supposer que l'ensemble des biens peuvent être traités comme des biens de première nécessité, dont la consommation est difficilement compressible, et qui de ce fait, soit ne sont pas substituables, soit le sont tellement qu'ils doivent être traités statistiquement comme un seul et même bien. On voit alors toute la logique sous-jacente au propos. L'hypothèse d'indépendance psychologique revient à adopter précisément un tel point de vue, c'est-à-dire à considérer que les biens servent tous des besoins différents (et qu'il n'existe pas véritablement de hiérarchie entre ces besoins, du moins au niveau collectif ?) ${ }^{44}$. Or c'est précisément par cette hypothèse que Allais justifie théoriquement l'essentiel de ses énoncés sur la demande.

Tout se passe donc comme si cette conséquence simplement vraisemblable venait justifier l'hypothèse très forte sur la distribution des caractéristiques des agents. Une fois encore, on ne peut manquer de souligner les libertés que Allais prend avec ses principes méthodologiques. Mais cette fois, la dynamique d'aller retour entre deux modes de validation, l'un fondé sur l'introspection, l'autre sur l'expérience des comportements économiques, ne fonctionne plus. Si la justesse supposée des conséquence vient légitimer la nature des hypothèses, nous avons quitté le cercle vertueux de construction théorique proposé au départ au profit d'une démarche beaucoup plus instrumentaliste.

Dans la discussion des lemmes 1 et 2, en revanche, l'usage de l'hypothèse d'indépendance psychologique est en quelque sorte inversé. Prise comme une donnée psychologique première à laquelle Allais associe des propriétés de marché non démontrées (sur les effets de revenu entre les différents marchés), l'hypothèse d'indépendance psychologique sert à justifier l'idée selon laquelle une dispersion raisonnable des préférences et des dotations serait suffisante pour garantir la stabilité. Cette fois, l'hypothèse d'indépendance psychologique est utilisée pour atténuer la charge de la preuve qui repose sur l'hypothèse de caractéristiques individuelles identiques. Ce dévoiement instrumentaliste, Allais semble en convenir de facto puisqu'il reconnaît par ailleurs que le système évoluera sans doute lentement vers un équilibre, du fait notamment que "les goûts sont très différents selon les individus" (Allais, 1993, 380).

Au final, toute la construction analytique qui conduit à la stabilité, dans le Traité, procède par accumulation d'approximations peu intuitives, il ne faut pas négliger son originalité. De par le lien qu'elle établit entre les hypothèses individuelles et la démonstration de la stabilité, elle confère à l'étude de Allais une grande unité, comparable à celle qui sous-tend les Eléments d'économie politique pure de Walras (cf. Allais, 1943, 169 ; 1993, 169). Mais ce projet, bien sûr, n'échappe pas à des limites qui renforcent un peu plus le sentiment d'un décalage entre les déclarations de méthode et leur mise en pratique. On sait bien par exemple que les fonctions

44 "En fait, les recherches statistiques effectuées sur les services de première nécessité montrent que dans le domaine très étendu où l'on a pu observer leur prix le coefficient d'élasticité est inférieur à un. Suivant nos définitions leur demande est donc inélastique et leur sensibilité positive. Ainsi pour les services de première nécessité, la valeur de la demande croît lorsque le prix croît. (...) De ces observations on peut déduire avec une probabilité très grande que dans la plus grande partie du domaine de variation du prix, il y a croissance de la valeur de la demande; autrement dit, $a_{2}$ est bien plus voisin de $a_{1}$ que de zéro. Nous verrons qu'il est possible de confirmer cette circonstance théoriquement" (Allais, 1993, 391) 
d'utilité additivement séparables n'ont pas d'implications simples sur les fonctions de demande excédentaires, à la différence par exemple des fonctions Cobb Douglas (voir Varian, 1985). Encore une fois, cette lecture est justifiée par les principes méthodologiques de Allais, qui garde à l'esprit, envers et contre tout, cet objectif de concilier réalisme et rigueur.

Finalement, l'hypothèse d'indépendance psychologique est doublement instrumentée :

En un premier sens, elle est instrumentée parce qu'elle permet de reproduire des énoncés d'expérience jugés vraisemblables et généraux.

En un second sens, parce qu'elle "réduit" la nature des hypothèses nouvelles (et des configurations) qu'il a fallu ajouter autrement pour parvenir à une démonstration complète de la stabilité.

Quoi qu'il en soit de la rigueur de la démonstration au regard des critères modernes ${ }^{45}$, on s'aperçoit que la stabilité est associée, pour Allais, à la possibilité de négliger les relations de dépendance psychologique extrême (qu'il s'agisse des complémentaires ou des substituts accusés). Son raisonnement sur la stabilité est donc ancré dans une représentation des dépendances psychologiques qui détermine étroitement les comportements de demande individuels et collectifs. Dans cette mesure, loin de donner une représentation abstraite de la stabilité économique, il l'intègre au contraire à une description du système économique en termes de données psychologiques, qu'il considère fondamentalement comme une source de son intelligibilité.

\section{Conclusion}

Au terme de ce parcours dans l'analyse et la méthodologie du Traité d'économie pure de Maurice Allais, on peut faire trois remarques conclusives.

D'un point de vue analytique, il n'y a pas de lien direct, encore moins de relation nécessaire ou suffisante, entre l'hypothèse d'indépendance psychologique et celle de stabilité. En revanche, sous la contrainte méthodologique que Allais se donne, qui consiste à rendre compte d'un ensemble varié de comportements, l'hypothèse d'indépendance psychologique est primordiale et conditionne par la suite, le choix des hypothèses complémentaires et les marges d'interprétation que l'on peut en donner dans la démonstration de la stabilité d'un équilibre général walrasien, et cela seul suffit à établir sa portée méthodologique et analytique.

Que reste-t-il finalement de la substituabilité, au terme de ce parcours ? Elle ne disparait pas, mais elle est limitée aux formes que permet l'usage d'une fonction d'utilité additive et séparable. La substitution entre les biens reste centrale, mais elle prend des formes modérées, créant les conditions pour que les biens soient

\footnotetext{
45 Selon Weintraub, "Ce qui reste à montrer, cependant, est que tous ces ajustements peuvent avoir lieu simultanément en ce sens que l'ajustement sur le marché $j$ en réponse au déséquilibre sur le marché $i$ ne va pas conduire, en fait, à une perturbation supplémentaire sur le marché $i$ qui en amplifierait la déviation par rapport à l'équilibre. Cette étape n'est pas franchie" (Weintraub, 1991b, 390). Il faut bien comprendre le sens d'une telle critique. Le fait que Allais adopte un raisonnement à la Walras, où les marchés s'équilibrent successivement et où la stabilité dépend de l'ampleur relative des effets directs et indirects de la variation des prix, ne suffit pas à affirmer que Allais n’a pas vu les difficultés liées à l'interdépendance des marchés. On remarquera d'ailleurs que Allais précise que les différents marchés jouant un rôle symétrique dans sa démonstration, indiquant par là que les effets de la variation du prix sur les autres marchés se confortent mutuellement dans le sens d'une réduction de la valeur de la fonction caractéristique (On trouvera un traitement de cette question dans Uzawa (1960)). Pour autant, il ressort bien de la présentation de Weintraub (1991a, 81-87) que l'hypothèse de substituabilité brute ne joue pas, à elle seule, un rôle comparable à celui que lui donnera la démonstration de Arrow, Block et Hurwicz (1959). Les lemmes 1 et 2 introduisent notamment des relations en valeur qui sont plus contraignantes et réduisent la généralité de la démonstration autant que sa structure.
} 
dans un relation symétrique (absence de substitution hiérarchique) qui sera elle aussi centrale pour théoriser les répercussions de la variation de prix d'un bien sur les autres marchés. Tant qu'elle n'est pas trop forte et qu'elle ne traduit pas de relations hiérarchiques entre les biens, la substituabilité est vertueuse dans l'esprit de Allais, et l'hypothèse d'indépendance psychologique représente bien ces propriétés aux yeux de Allais.

Enfin, l'entreprise originale et largement solitaire de Allais fait écho aux développements parallèles de l'analyse de la stabilité de Hicks (1939) d'une manière qui nous semble particulièrement frappante. Dans les deux cas, la représentation et la justification des propriétés de stabilité d'une économie walrasienne abstraite repose sur l'idée que les comportements "réels" ou "vraisemblables" de demande agrégés sinon individuels exhibent globalement certaines propriétés de substitution qui suffisent à garantir la stabilité. Dans la tradition hicksienne proprement dite, ces propriétés s'expriment directement dans le langage moderne de la substituabilité issu de Slutsky, et donne lieu à des justifications qui font intervenir des hypothèses sur la répartition des effets de revenu. Chez Allais, les propriétés agrégées des demandes excédentaires sont justifiées également par des hypothèses qui reviennent à négliger les effets de répartition dus aux variations du système de prix (identité des goûts et des revenus), mais au-delà, par des hypothèses qui visent à ancrer le comportement de demande dans des déterminations psychologiques. L'intérêt et l'originalité de cette construction vient de ce qu'elle fait appel non seulement à la distribution des revenus mais aussi à celle des préférences, l'une comme l'autre étant supposées très resserrées autour d'une norme, un homme moyen. Plutôt que d'en appeler a priori à la faiblesse et à la symétrie des effets de revenus, Allais privilégie là encore d'en donner une justification à partir des distributions des caractéristiques individuelles. Il est tentant de faire un rapprochement avec les résultats récents de la théorie de la demande et de l'agrégation. L'hypothèse très restrictive sur la distribution des préférences et des revenus laisse entrevoir les difficultés d'un fondement microéconomique des fonctions de demande excédentaire ayant de bonnes propriétés (SonnenscheinMantel-Debreu) ${ }^{46}$. Mais tandis que les théorèmes des années 70 s'inscrivent dans un climat pessimiste, chargé de la connaissance des travaux sur la stabilité des années 50 et 60 , la proposition de Allais se veut tout à fait rassurante ; elle se place dans la perspective optimiste des fondements microéconomiques.

\section{RÉFÉRENCES BIBLIOGRAPHIQUES}

Allais M. (1943) A la recherche d'une discipline économique. Première partie: l'économie pure, Ateliers Industrie, réédité sous le titre Traité d'économie pure, Imprimerie Nationale et Centre national de la Recherche Scientifique, Paris, 1953.

Allais M. (1978) Contributions à la science économique : vue d'ensemble 1943-1978; Paris : Centre d'Analyse Économique

ALLAIS M. (1988) "la théorie du choix dans l'œuvre de René Roy, une analyse critique", Revue d'économie politique, 98(3), mai-juin 1988, 315-57.

AlLAIS M. (1989) 'La philosophie de ma vie", Revue d'économie politique, 99(1), 28-54.

Allais M. (1993) Traité d'économie pure, Paris, éd. Clément Juglar, 15-155

46 En s'appuyant sur des hypothèses en termes de distribution, Allais paraît anticiper sur la démarche d'Hildenbrand (1994). Là encore, il ne faut pas se méprendre, Allais ne cherche pas à créer de la structure au travers de l'agrégation, il cherche simplement à la conserver. D'où son insistance sur la concentration des individus autour de caractéristiques identiques. Mais ici, la perspective d'une construction unifiée allant des données psychologiques individuelles aux propriétés d'une économie d'échange fait ressortir, plus crûment que chez Hicks (1939), la nécessité d'imposer des restrictions fortes sur les caractéristiques individuelles. 
AlLais M. (1997) "An Outline of My Main Contributions to Economic Science", American Economic Review, 87(6), 3-12.

Allen R. G. D. (1934) “A Comparison between Different Definitions of Complementary and Competitive Goods", Econometrica, 2, 168-75.

Arrow K. J., H. D. BlOCK et L. HurwiCZ (1959) "On the Stability of the Competitive Equilibrium II", Econometrica, 27, 82-109.

ArRow K. J. et L. HurWICZ (1958) "On the Stability of Competitive Equilibrium I", Econometrica, 26, 522-52.

Belloc B. et MoreauX M. (1987) "Allais, Maurice", in J. Eatwell, M. Milgate et P. Newman (eds) The New Palgrave: A Dictionary of Economics, London Macmillan, 1987, vol.1, 78-9.

Boiteux M., de Montbrial T. et Munier B. (1986) Marchés, Capital et Incertitude. Essais en l'honneur de Maurice Allais, Paris, Economica.

Bousquet G. (1930) Institutes de science économique, t.1: Introduction à la science économique, Paris, Rivière.

CHAIGNEAU N. (1998) Contrat et utilité : origines et fondements de la théorie de l'échange de Francis Y. Edgeworth, Thèse, Paris I.

Chaigneau N. et LE Gall Ph. (1998) "The French Connection: The pioneering econometrics of Marcel Lenoir", in W. J. Samuels (ed.), european economists of the Early 20th Century, Vol. 1. Studies in Neglected Thinkers of Belgium, France, The Netherlands and Scandinavia, Edward Elgar, 163-189

ChARrEtTE, L. et C. BRONSARD (1975) “Antonelli-Hicks-Allen et Antonelli-Allais-Barten. Sur l'utilisation des conditions d'intégrabilité d'Antonelli", Recherches économiques de Louvain, 41 (1), mars, 25-34.

CHIPMAN J. (1956) “Review of Maurice Allais' Traité d'économie pure”, Kyklos, 1956, 9 (4), 513-515

CHIPMAN J. (1977) "An Empirical Implication of Auspitz-Lieben-Edgeworth-Pareto Complementarity ", Journal of Economic Theory, 14 (February), 229-231

Chipman J. et LENFANT J-S. (2002) “Slutsky's 1915 Article : How It Came to Be Found and Interpreted ", History of Political Economy, 34(3), 553-597

COLSON C. (1924) Cours d'économie politique, Livre premier: Théorie générale de phénomènes économiques, édition définitive (première édition en 1915), Paris, Gauthier-Villars et $C^{i e}$.

DeBreu G. (1974) "Excess Demand Functions", Journal of Mathematical Economics, vol.1, 15-23.

DIVISIA F. (1928) Economique rationnelle, Paris, Doin.

DrEzE J. H. (1989) “Maurice Allais and the French Marginalist School”, Scandinavian Journal of Economics, 91(1), 5-16.

FISHER I. (1892) Mathematical Investigations in the Theory of Value and Prices. New Haven: Connecticut Academy of Arts and Sciences, Transactions 9. Reprinted, New York: Augustus M. Kelley, 1961.

FRIEDMAN M. (1934) The fitting of indifference curves, Manuscript.

GRANDMONT J.-M. (1989) "Rapport sur les travaux scientifiques de Maurice Allais", Annales d'Economie et de Statistiques, 14, 25-38.

HAYEK F. (1943) "The Geometrical Representation of Complementarity", The Review of Economic Studies, 10:2, 122-5.

Hicks J. R. (1937) Théorie mathématique de la valeur en régime de libre concurrence, Paris, Hermann \& $C^{\circ}$.

HiCKS J. R. (1939) Value and Capital, Oxford at the Clarendon Press, 2e édition 1946.

Hildenbrand W. (1994) Market Demand: Theory and Empirical Evidence, Princeton, Princeton University Press.

JOHnson W. E. (1913) "The Pure Theory of Utility Curves", Economic Journal, 23, repris in W. J. Baumol et S. N. Goldfeld (eds.), Precursors in Mathematical Economics : An Anthology. London : London School of Economics and Political Science, 1968, 97-117.

LYAPOUNOV A. (1907) "Problème général de la stabilité du mouvement", Annales de la Faculté des Sciences de l'Université de Toulouse, 9, 203-475.

LENOIR M. (1913) Etudes sur la formation et le mouvement des prix, Paris : Giard et Brière.

MANTEL R. (1974) "On the Characterization of Aggregate Excess Demand Functions", 
Journal of Economic Theory, 7 (March), 348-353.

Munier B. (1986) "L'œuvre d'économiste de Maurice Allais", in Boiteux, de Montbrial et Munier, 13-37.

Munier B. (1989), "Portée et signification de l'œuvre de Maurice Allais, Prix Nobel d'Economie 1988 ", Revue d'économie politique, 1-27.

NEGISHI T. (1958) "A Note on the Stability of an Economy where all Goods are Gross Substitutes", Econometrica, 26, 445-7.

Negishi T. (1962) "The Stability of a Competitive Economy: A Survey Article", Econometrica, 30, 635-69.

Pareto V. (1909) Manuel d'économie politique, Droz, Genève-Paris, 1981.

RoY R. (1942) De l'utilité - contribution à la théorie des choix, Paris, Hermann.

SAMUELSON P. A. (1938a) "A Note on the Pure Theory of Consumer's Behaviour", Economica, N.S., 5, 61-71. "An Addendum” Ibid., 5, 353-4.

SAMUELSON P. A. (1938b) "The Empirical Implications of Utility Analysis", Econometrica, 6, 344-56.

SAMUELSON P. A. (1941) "The Stability of Equilibrium: Comparative Statics and Dynamics", Econometrica, 9, 97-120.

SAMUELSON P. A. (1942) "The Stability of Equilibrium : Linear and Nonlinear Systems", Econometrica, 10, 1-25.

SAMUELSON P. A. (1947) The Foundations of Economic Analysis. Cambridge, enlarged edition, Mass. : Harvard University Press, 1983.

SCHultz H. (1938) The Theory and Measurement of Demand, Chicago. University of Chicago Press.

SONNENSCHEIN H. (1973) "Do Walras' Identity and Continuity Characterize the Class of Community Excess Demand Functions ?”, Journal of Economic Theory, vol.6, 34554.

VARIAN H. (1985) "Additive Utility and Gross Substitutes", Mimeo, University of Michigan, Ann Arbor

WALRAs L. (1874-1926) Eléments d'Economie Politique Pure (ou théorie de la richesse sociale), vol. VII des CEuvres économiques complètes d'Auguste et Léon Walras, Paris, Economica, 1988.

WALRAS L. (1900) Abrégé des Eléments d'économie politique pure, in Walras (1874-1926), 739791.

WeBER C. (1999) "Slutsky and Additive Utility Functions, 1947-1972", History of Political Economy, 31(2), 393-416

Weber C. (2000) "Two Further Empirical Implications of Auspitz-Lieben-EdgeworthPareto Complementarity ", Economic Letters, 67, 289-95.

Weintraub E. R. (1991a) Stabilizing dynamics. Constructing economic knowledge, Cambridge, Cambridge University Press.

Weintraub E. R. (1991b) "Allais, Stability, and Liapounov Theory", History of Political Economy, 23 (3), 383-418. 